\title{
Meta-Context and Choice-Set Effects in Mini-Dictator Games
}

Citation for published version (APA):

Panizza, F., Vostroknutov, A., \& Coricelli, G. (2019). Meta-Context and Choice-Set Effects in Mini-Dictator Games. Maastricht University, Graduate School of Business and Economics. GSBE Research Memoranda No. 010 https://doi.org/10.26481/umagsb.2019010

Document status and date:

Published: 16/04/2019

DOI:

10.26481/umagsb.2019010

Document Version:

Publisher's PDF, also known as Version of record

\section{Please check the document version of this publication:}

- A submitted manuscript is the version of the article upon submission and before peer-review. There can be important differences between the submitted version and the official published version of record.

People interested in the research are advised to contact the author for the final version of the publication, or visit the DOI to the publisher's website.

- The final author version and the galley proof are versions of the publication after peer review.

- The final published version features the final layout of the paper including the volume, issue and page numbers.

Link to publication

\footnotetext{
General rights rights.

- You may freely distribute the URL identifying the publication in the public portal. please follow below link for the End User Agreement:

www.umlib.nl/taverne-license

Take down policy

If you believe that this document breaches copyright please contact us at:

repository@maastrichtuniversity.nl

providing details and we will investigate your claim.
}

Copyright and moral rights for the publications made accessible in the public portal are retained by the authors and/or other copyright owners and it is a condition of accessing publications that users recognise and abide by the legal requirements associated with these

- Users may download and print one copy of any publication from the public portal for the purpose of private study or research.

- You may not further distribute the material or use it for any profit-making activity or commercial gain

If the publication is distributed under the terms of Article $25 \mathrm{fa}$ of the Dutch Copyright Act, indicated by the "Taverne" license above, 


\title{
Meta-Context and Choice-Set Effects in Mini-Dictator Games*
}

\author{
Folco Panizza $^{\dagger} \quad$ Alexander Vostroknutov ${ }^{\ddagger} \quad$ Giorgio Coricelli ${ }^{\Uparrow}$
}

April 5, 2019

\begin{abstract}
Knowing that some action is possible in principle, even if not available, could affect behaviour. This may happen because a game is perceived as part of a larger game or 'metacontext' that includes its outcomes as a proper subset. In an experiment we test the effects of meta-context and specific choice sets on pro-social behaviour in a series of binary miniDictator games by eliciting participants' normative evaluations, fitting a norm-dependent utility, and analysing the residuals. We find that participants' normative evaluations in miniDictator games derive from the meta-context (a standard Dictator game) and explain a sizeable portion of variance in choices. Restricted choice sets of mini-Dictator games also influence participants' decisions: they take into account dictator's losses and recipient's gains from choosing the prosocial action as fractions of their respective maximum payoffs. This choice-set effect correlates with individual measures of rule-following propensity supporting the idea that it is also normative. Thus, there are two types of normative reasoning that contribute to pro-social behaviour: a meta-context and a choice-set effect.
\end{abstract}

JEL classifications: C91, C92, D91.

Keywords: mini-Dictator games, meta-context, choice-set effects, norms, norm-dependent utility.

*We would like to thank the participants of the Florence-Konstanz Workshop on Behavioral Social Sciences (Nov 2017) and seminars in Vienna Center for Experimental Economics (Oct 2017) and Max Planck Institute for Research on Collective Goods (May 2017) for invaluable comments. GC acknowledges the financial support of the European Research Council (ERC Consolidator Grant 617629). All mistakes are our own.

${ }^{\dagger}$ Center for Mind/Brain Sciences, University of Trento, Via delle Regole 101, 38123 Mattarello (TN), Italy. e-mail: folco.panizza@unitn.it

${ }_{\ddagger}^{\ddagger}$ Department of Economics (AE1), Maastricht University, Tongersestraat 53, 6211LM Maastricht, The Netherlands. e-mail: a.vostroknutov@maastrichtuniversity.nl

$\S$ Corresponding author.

IDepartment of Economics, University of Southern California, 3620 South Vermont Ave. 300, Los Angeles, CA 90089, USA. 


\section{Introduction}

The history of thinking about the nature of social behaviour in games can be seen in terms of the scope in which players in a game operate. In early days of game theory it was common to make the simplest assumption that players are maximizing their own self-interest (Morgenstern and Von Neumann, 1953). However, with the rise of experimental economics, a lot of evidence has emerged that challenged this hypothesis. Frameworks like Kahneman, Knetsch, and Thaler's Dictator game (Kahneman et al., 1986) have contributed to the formulation of alternative models collectively known as social preferences (e.g., Fehr and Schmidt, 1999; Bolton and Ockenfels, 2000). These models introduced the idea that, to explain deviations from the self-interest, the utility of an outcome should also include the payoffs of other individuals obtained in this outcome. Thus, the 'scope of operation' has been enlarged to include 'universal' considerations about others players' payoffs like inequity aversion. This process, however, did not stop there. Later experimental studies have shown the susceptibility of participants to experimental cues or context. Dictator game experiments that manipulated anonymity (Hoffman et al., 1996; Franzen and Pointner, 2012) or choice set (List, 2007; Bardsley, 2008; Cappelen et al., 2013) have shaped the notion that the utility, while still seemingly modulated by other players' payoffs, also depends on the context of a game. For example, List (2007) and Bardsley (2008) show that providing dictators with the possibility to take money from the recipients makes them move away from the half-half split towards more selfish options, as if the complete set of possible outcomes played a role in participants' decisions. These results enlarged the scope even further by showing that universal social preferences could not account for context effects and that the characteristics of a game at hand needed to be taken into account. The challenge of incorporating context was taken by the social norms literature where players maximize norm-dependent utility (Cappelen et al., 2007; López-Pérez, 2008; Krupka and Weber, 2009, 2013; Kessler and Leider, 2012; Kimbrough and Vostroknutov, 2016). Norms represent the rules of social conduct that prescribe the appropriateness of each action in a given setting. To the extent that the cues about an environment also inform about the norms, norm-dependent models have been able to explain the differences in behaviour due to context effects. ${ }^{1}$

The focus of some studies in this line of research is on the context meant as a frame in which the game is presented in the instructions (e.g., Liberman et al., 2004; Lightner et al., 2017; Chang et al., 2018; Kimbrough et al., 2018). Other studies manipulated the set of options in the Dictator game (DG), as in the above mentioned experiments, or the dictator's and recipient's initial endowments (e.g., Cox et al., 2016). While the meaning of context varies widely across all these studies, it is generally limited to the game played at the moment: participants are presented with one situation, and the influence of context is measured between-subjects.

\footnotetext{
${ }^{1}$ Social norms literature does not claim that people do not have social preferences, but rather that social preferences emerge as a consequence of following norms (Kimbrough and Vostroknutov, 2016).
} 
What is missing in this conceptualisation, and what we want to address in our experiment, is that a game can be perceived as being part of a larger game that includes its outcomes as a proper subset. Knowing that some action is possible in principle, even if there is no possibility to take this action, could affect behaviour. In other words, a game can be perceived in terms of a 'meta-context' or a supergame that includes the current one, and, thus, can influence decisions. We conjecture that what is getting transferred from the meta-context are the norms, which define the appropriateness of actions in the game that is actually being played. ${ }^{2}$ Some support for this hypothesis comes from Thomsson and Vostroknutov (2017), where a 'constrained' DG is studied in which dictators cannot give more than half of their endowment to the recipient. The results show that dictators believe that equal split is the most appropriate action, as if they are considering the whole dictator game without constraints, which is also reflected in their behaviour. ${ }^{3}$ Chlaß and Moffatt (2012) study choices in a sequence of Dictator games with varying choice sets. The authors find that, if in the first game participants are given an additional option to take from the recipient, the amount given in subsequent games is less likely to change (even when this take option is absent) than in a treatment in which this option is only introduced in later games. This suggests that participants use the early option to take as a reference for the subsequent games, thus creating a meta-context.

Despite our thinking that meta-context influences the behaviour in social situations, we do not believe that it is the only important factor. In particular, we propose that, in addition to meta-context, the specific choice set of a single game may also affect decisions. In fact, in order to detect any choice-set effects, we need meta-context as a benchmark, since otherwise it would be impossible to tell what is a choice-set effect and what is not. ${ }^{4}$ Therefore, the second goal of this study is to explore whether and in what way a restricted choice set (compared to the supergame) can influence dictator giving. We are, of course, not the first to think about this problem. The studies mentioned above are, to an extent, concerned with the comparisons of Dictator games with varying sets of options. However, unlike these studies where two or three treatments are contrasted against each other, we aim at uncovering the systematic relationships between the al-

\footnotetext{
${ }^{2}$ We do not consider the possible effects of social preferences, since these models are based on outcomes rather than contextual information. This means that meta-context is irrelevant for the calculation of social utility (see Section 4 for discussion).

${ }^{3}$ Note that the subjects could have thought about the constrained DG with dictator's endowment $X$ (where they cannot give more than $X / 2$ ) as a DG with endowment $X / 2$ plus a 'gift' of $X / 2$ to the dictator. In this case it is reasonable that they should consider sending $X / 4$ as a socially appropriate choice. However, a considerable fraction of subjects still chooses to send the amounts close to $X / 2$, as Thomsson and Vostroknutov (2017) report. Interestingly, List (2007) studies a DG where subjects are told that they receive $X / 2$ for themselves and another $X / 2$ that they can share with the recipient. In this case few dictators send more than $X / 4$. This shows that meta-context reasoning can be induced by simply manipulating instructions.

${ }^{4}$ We could have used social preferences in place of meta-context. However, as we discussed above, social preferences cannot account for any context effects, thus, what we could achieve at best is a result that, in some specific supergame, there are some choice-set effects. This, though interesting, would not be generalisable to other environments.
} 
locations available in the restricted choice sets and the observed behavioural changes that could be generalised beyond Dictator games.

Our experiment is designed as follows. We employ an extensive within-subject design in which participants choose in a series of two-alternative mini-Dictator games (mini-DGs) with all allocations of payoffs for a dictator and a recipient drawn from the 'supergame' set $\left\{\left(\pi_{d}, \pi_{r}\right) \mid \pi_{d}, \pi_{r} \geq\right.$ 0 and $\left.\pi_{d}+\pi_{r}=60\right\}$. In other words, dictators always choose between two alternatives that lead to some divisions of 60 tokens (without the possibility of taking money from the recipient). Next, we use the task proposed by Krupka and Weber (2013) to elicit norms from the same participants in a subset of these mini-DGs. Finally, we estimate the propensity to follow rules by means of a rule-following task (Kimbrough and Vostroknutov, 2018b). In order to test the meta-context hypothesis, that participants derive the appropriateness of actions in mini-DGs to some extent from the standard DG (the meta-context), we check if the norms, elicited in mini-DGs, reflect the normative values generally obtained from the same task in the standard DG (e.g., Krupka and Weber, 2013; Kimbrough and Vostroknutov, 2016, 2018b). If the meta-context hypothesis holds, we should observe some consistency between the norms elicited in mini-DGs and the norms elicited in the standard DG. In order to disentangle choice-set effects from the meta-context we should consider two possibilities: 1) the elicited norms do reflect choice-set dependence, in which case we can use the elicited norms to detect choice-set-dependent and choice-set-independent components; 2) the elicited norms do not reflect choice-set dependence, in which case we use the variance in the choice data, unexplained by the norm-dependent utility, to estimate choice-set effects. In both cases, we use the subject-specific normative evaluations to fit the parameters of a norm-dependent utility to the choice data and check how well the elicited norms can explain the behaviour.

The data from the norm-elicitation task confirm the meta-context hypothesis: participants do perceive the norms in binary mini-DGs as derived from a supergame. Interestingly, the normative ratings are so consistent with those in a standard DG, that we detect no choice-set dependence at all. Thus, participants express clear meta-context norms, which are not influenced by the choice set of the mini-DGs. The estimation of the norm-dependent utility shows that these normative ratings explain a sizeable portion of variance in choices. This being said, we also find that for a half mini-DGs the norm-dependent utility fails to completely explain the behaviour. The discrepancy in predictions is the largest for the mini-DGs in which the dictator receives very high payoffs in both allocations and recipient receives very low payoffs. Notably, the participants are more generous in these situations than the norm-dependent utility predicts. We show that, in order to account for this behaviour, we need to assume that participants care about the cost of choosing a non-selfish option, measured in percentages of their wealth, relative to the gain of the recipient, measured in percentages of her wealth. Put simply, participants are more generous when they lose relatively little in comparison with a large increase in recipients' wealth. Thus, we identify a very specific type of choice-set effect that works on top of the meta- 
context. ${ }^{5}$ Our last finding concerns the nature of the choice-set dependency. We find that its strength is correlated with the individual measures of rule-following propensity, thus, supporting the hypothesis that choice-set effects are normative and do not operate through a separate channel, despite not being detected by the norm-elicitation task.

Our findings demonstrate that context can influence social decisions in a rather complex way. In particular, there are two types of normative reasoning that contribute to pro-social choices in a given situation. One is the 'absolute' normative component that comes from the meta-context and is independent from the choice set of a specific game. It determines how the appropriateness of an allocation is viewed on a larger scale irrespective of the specifics of choices involved. Another is the 'relative' normative component that elaborates on the first one by taking the available alternatives into account. Both components contribute substantially to the incentives that drive pro-social behaviour.

\section{Experimental Design}

The experiment consisted of three decision-making stages: the Rule Following (RF) task, the series of mini-Dictator games, and the norm-elicitation task.

\subsection{The Rule-Following Task}

In the rule-following task (Kimbrough and Vostroknutov, 2018b), participants decided how to allocate 50 balls between a blue and a yellow bucket. The position of the two buckets was randomised across individuals. Participants earned $€ 0.05$ for each ball they dropped in the blue bucket, and $€ 0.10$ for each ball they dropped in the yellow bucket. The instructions explicitly stated that 'the rule is to put the balls into the blue bucket.' This payment scheme and the rule were the only information provided to participants. The total payoff in this stage was the sum of earnings from both buckets. Therefore, the amount of money earned could vary from $€ 2.50$ (always following the rule) to $€ 5.00$ (never following the rule).

The RF task creates a situation in which participants are asked to follow an arbitrary rule that decreases their payoff and yet entails no cost of breaking it. This allows us to measure the propensity of participants to stick to a non-social rule that, as was demonstrated by Kimbrough and Vostroknutov (2016), also predicts pro-social behaviour.

\subsection{The Mini-Dictator Games}

In Stage 2, participants played a series of mini-Dictator games with constant sum of payoffs. In each game each participant chose between two divisions of 60 tokens $(1$ token $=€ 0.10)$ split

\footnotetext{
${ }^{5}$ To make sure that we are dealing with the choice-set effects and not with a misspecification of an outcome-based utility model, we estimate several other outcome-based models. None of them reconciles the observed behaviour.
} 
between her and an unknown other. 27 different allocations were used in all mini-DGs, including an equal split. Out of them, 13 allocations were advantageous to the dictator (she received more than the recipient), and 13 allocations were disadvantageous (the dictator received less than the recipient). The allocations were combined to form a mini-DG according to the two criteria: 1) one of the two allocations has to be equal split or advantageous to the dictator; 2) if one allocation is disadvantageous, it has to be less unequal than the other (advantageous) allocation. These criteria yielded a total of 182 mini-DGs. The list of all mini-DGs can be found in Table 7, Appendix B. Mini-DGs were presented to each participant in an individually generated random order.

After the task was completed, participants were randomly paired and one of them was selected as a dictator. Consequently, one of the choices of this individual was randomly implemented. Participants were fully informed about all these procedures.

\subsection{Norm Elicitation}

In order to elicit participants' beliefs about norms we used the norm-elicitation task proposed by Krupka and Weber (2013). Participants were presented with a selection of 18 mini-DGs that they encountered in the previous task. They rated on a 4-point Likert scale the degree of social appropriateness of picking each alternative in each game. Each mini-DG was presented on a separate screen, and the order of their appearance was randomised. To detect choice-set effects, half of the allocations were repeatedly presented in combination with different alternatives. The list of all mini-DGs used for norm elicitation can be found in Appendix A Table 4.

To incentivise precise answers, participants were rewarded by means of a coordination game. One option in one of the mini-DGs was randomly selected. If participant's rating matched that of the majority in the session, he/she received $€ 5.00$.

\subsection{Subjects}

Participants were recruited from the subject pool of the Cognitive and Experimental Economics Laboratory at the University of Trento and invited via e-mail. 166 subjects (93 female, mean age $=22$ ) completed the experimental task. The study was approved by the University Ethical Committee. Experiment was programmed in z-Tree (Fischbacher, 2007). Experimental sessions were run in May 2017, February and March 2018. There were no pilots, and no data were discarded. 


\section{Results}

\subsection{Summary Results}

We start with reporting some summary results in order to show consistency between the measures of rule-following and behaviour in mini-DGs. Figure 1A shows the histogram of rulefollowing propensity estimated by the RF task. Consistent with findings in different countries (Kimbrough and Vostroknutov, 2018b), there are participants who are strong rule-followers, strong rule-breakers, or have intermediate level of rule-following propensity. The proportion of strong rule-followers, who put all the balls in the blue bucket, is $20 \%$, whereas the proportion of strong rule-breakers (all balls in the yellow bucket) is $13 \%$.
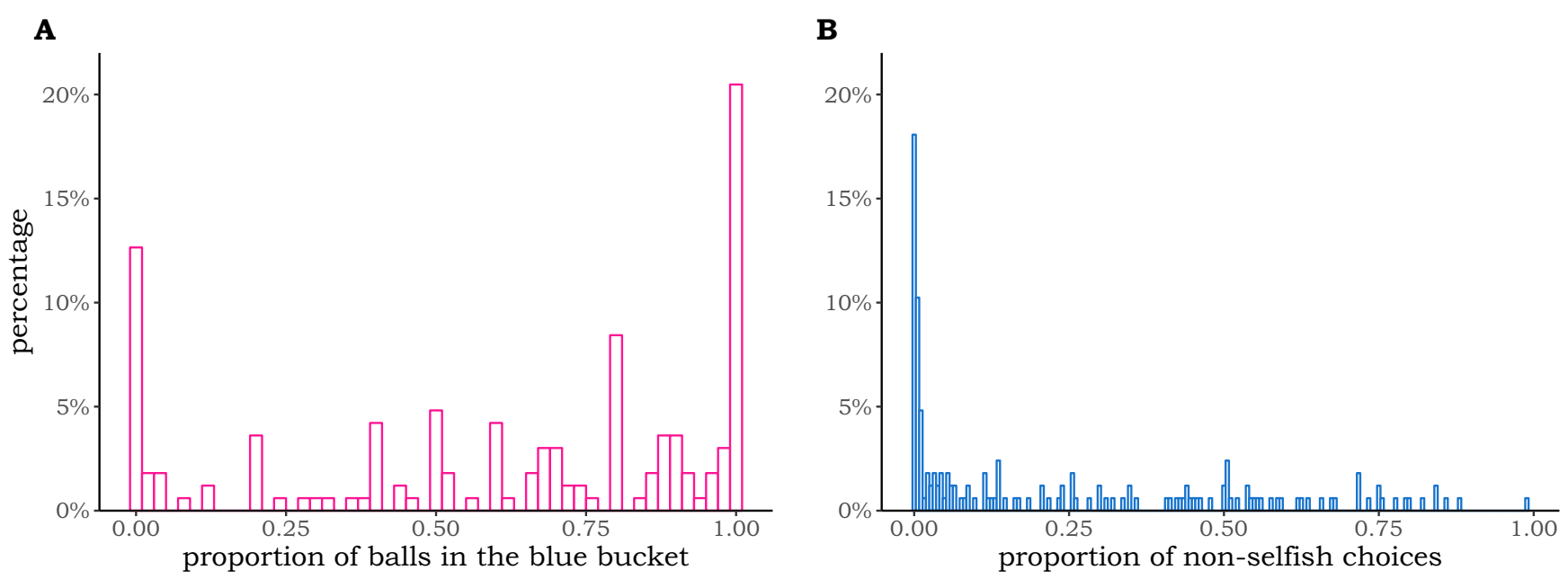

Figure 1: A. Histogram of the propensity to follow rules as measured by the proportion of balls in the blue bucket in the RF task. B. Histogram of the proportion of non-selfish choices in the mini-DGs.

Next, we look at the aggregate behaviour of participants in the mini-DGs. We calculate for each participant the proportion of non-selfish choices (participant chooses the allocation that gives her less payoff than the alternative). Figure 1B shows the histogram. These results cannot be directly compared to the standard Dictator game, as the choices in our task are between two allocations, and we employ multiple games rather than a single one. Hutcherson et al. (2015) and Kuss et al. (2015) use mini-DG tasks that are similar to ours. The two papers report the proportions of $21 \%(\mathrm{sd}=18 \%)$ and $13.2 \%$ of non-selfish choices respectively. Our results are in line with these findings if not slightly higher, with an average of $23.9 \%$ ( $\mathrm{sd}=27.3 \%$ ) of non-selfish choices. Notice that more than half of the participants made at least $10 \%$ of non-selfish choices, and these choices are more likely when the cost of taking them is small (logistic regression of absolute difference in own payoffs on the probability of choosing a non-selfish option, $Z=4.7$, $p<.001)$. This shows that participants do respond to standard economic incentives. 
We observe that the behaviour is very heterogeneous in both the mini-DGs and the rulefollowing task. Thus, we explore the relationship between individual rule-following propensity and the proportion of non-selfish choices (Figure 8 in Appendix A shows the scatter plot). Notice that rule-breakers on average choose selfish allocations $97 \%$ of the time, whereas for rulefollowers this rate is only $58 \%$. Spearman's rank correlation shows that this relation is significant $(\rho=0.42, p<.001)$. This association between rule following and dictator giving confirms the findings of previous experiments (Kimbrough and Vostroknutov, 2016, 2018b) and provides a good indication that the behaviour in mini-DGs is related to participants' sensibility to norms.

\subsection{Meta-Context}

While the RF task is a simple measure of the propensity to follow non-social rules, behaviour in the Dictator game setting is arguably driven by social inputs. In order to understand, from the normative perspective, whether mini-DGs are considered as standalone games or as a part of a meta-context, we elicit the beliefs about socially appropriate behaviour using the normelicitation task. Figure 2 plots the appropriateness ratings of the 19 allocations presented during the task averaged across subjects. ${ }^{6}$ Mean, standard error, and median ratings for each allocation in each mini-DG are presented in Table 4 in Appendix A. It is clear that participants' ratings show the same pattern that emerges when the norms are elicited in standard DG, namely, the half-half allocation is considered the most appropriate, while the more unequal allocations are increasingly less appropriate. This demonstrates that, normatively, participants treat mini-DGs as a part of a meta-context, in this case standard DG, in which all actions are available. In support of this finding, notice that average ratings show little variability, which means that participants mostly agree on the appropriateness evaluations.

Despite the similarities with norm elicitations in standard Dictator game, we observe that the shape of the curve is slightly different. We expected to observe an asymmetry in ratings between advantageous (more money to oneself) and disadvantageous (more money to the other) splits, with the former being rated as less appropriate than the latter (Kimbrough and Vostroknutov, 2016; Krupka and Weber, 2013; Chang et al., 2018). This, however, is not the case, as the two types of splits are almost perfectly symmetric. Another aspect that differs from previous experiments is that the increase in appropriateness from less to more appropriate allocations is much less convex than in other studies. We do not have a precise explanation for these differences, but speculate that restricted choice set could have played a role.

We also check whether perception of appropriateness differs between rule-followers and rule-breakers. We divide the sample by median of rule-following propensity and run one Wilcoxon rank-sum test for each allocation presented, with participant's type as a grouping variable. Un-

\footnotetext{
${ }^{6}$ Some allocations were presented multiple times in pair with different allocations. In this plot we average across multiple presentations.
} 


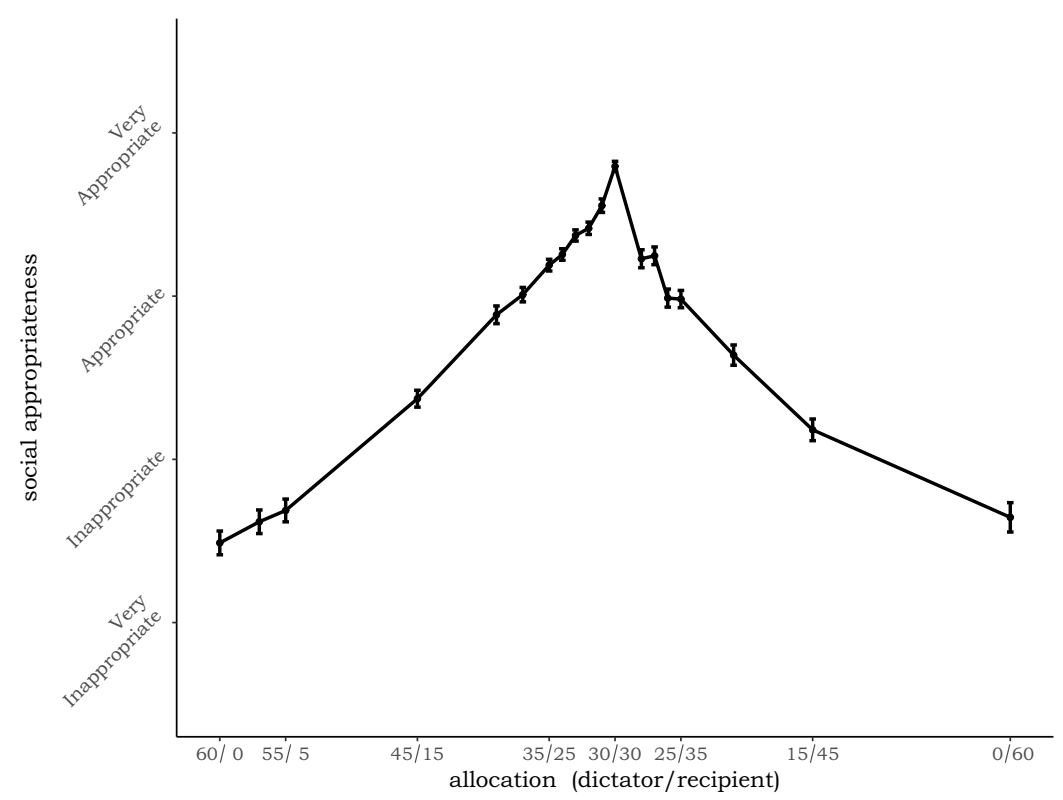

Figure 2: Average appropriateness ratings across allocations. On the $x$-axis: Dictator's payoff/Recipient's payoff.

like Kimbrough and Vostroknutov (2018b), we find that appropriateness ratings are not significantly different (all $p>.05$ ). This suggests that, while choices in the mini-DGs range from completely selfish to very generous, participants are all similarly aware of what the normative expectations are. Finally, we test for the presence of the choice-set effects, namely, that allocations, which were repeatedly presented in different mini-DGs, are rated differently depending on the alternative. None of the rating differences are significant even at the $10 \%$ level (KruskalWallis test).

All these findings taken together provide a strong evidence that participants derive normative values of allocations in mini-DGs from a single meta-context, which is independent of the allocations in specific games. We further discuss the implications of this result in Section 4.

\subsection{Norm-Dependent Utility Estimation}

The next step in our analysis is to estimate how well norm-dependent utility, which uses the information about elicited norms, fits the observed choices. Given that the appropriateness beliefs do not depend on the rule-following propensity or choice set, we use the ratings from the allocations in the norm-elicitation task to interpolate the appropriateness of all other dictator allocations in the mini-DGs. Having an appropriateness rating for all allocations would allow us to use them in a utility function. We estimate a 'norm function' as a piece-wise power curve for advantageous and disadvantageous allocations, fitted separately for each participant. The parameters $a_{a d v}, b_{a d v}, c_{a d v}, a_{d i s}, b_{d i s}, c_{d i s}$ of the power curves are estimated using non-linear fitting 
of the form:

$$
N\left(\pi_{d}, \pi_{r}\right)= \begin{cases}a_{a d v}+b_{a d v} \cdot E^{c_{a d v}}+\varepsilon_{a d v} & \text { if } \pi_{r} \geq 30 \\ a_{d i s}+b_{d i s} \cdot E^{c_{d i s}}+\varepsilon_{d i s} & \text { if } \pi_{r} \leq 30\end{cases}
$$

The dependent variable $N\left(\pi_{d}, \pi_{r}\right)$ represents the social appropriateness ratings of the 19 allocations. The values of $N\left(\pi_{d}, \pi_{r}\right)$ are in the set $\left\{-1,-\frac{1}{3}, \frac{1}{3}, 1\right\}$, where -1 stands for 'very inappropriate' and 1 for 'very appropriate.' The independent variable $E$ is a standardised measure that orders allocations by their equality, ranging from 0 (highest inequality, e.g., 60/0 or 0/60) to 1 (complete equality, e.g., 30/30). ${ }^{7}$ A parameter summary for the power curves (Table 5) together with a fitting example (Figure 9) are presented in Appendix A.

Next, we compare advantageous and disadvantageous curves at group level. Analysis of the power parameters $c_{a d v}$ and $c_{d i s}$ reveals that ratings for disadvantageous allocations are more convex (i.e., differences in appropriateness are more accentuated) than for advantageous allocations (median $c_{d i s}=2.29$, median $c_{a d v}=1.49$; Wilcoxon signed rank test with continuity correction, $V=2927, p<.001)$. This difference means that as the disadvantageous options become more unequal, they decrease in appropriateness faster than advantageous options. This is in line with observations we made in regard to Figure 2. Neither $a_{a d v}, a_{d i s}$ nor $b_{a d v}, b_{d i s}$ differ significantly between the two allocation types.

With an appropriateness rating for each allocation at hand, we estimate a utility function for participants' choices. We model the utility of participant $i$ using the specification by Kessler and Leider (2012): $U_{i}\left(\pi_{d}, \pi_{r}\right)=\beta_{i} \pi_{d}+\gamma_{i} \cdot N_{i}\left(\pi_{d}, \pi_{r}\right)$. This model assumes that participants derive utility from their own gains (first term) and from the norm compliance (second term). The normative component of the utility is a norm function weighed by $\gamma_{i}$, which defines the propensity of the participant to follow the social norm. We estimate parameters $\beta_{i}$ and $\gamma_{i}$ by means of a logistic regression assuming random utility, namely that the probability of choosing the option with the highest utility is proportional to the utility difference between the two options (McFadden, 1976). We estimate the following regression:

$$
\operatorname{Pr}(\text { non-selfish choice })=\alpha_{i}+\beta_{i} \cdot\left(\pi_{d}^{\text {nonself }}-\pi_{d}^{\text {self }}\right)+\gamma_{i} \cdot\left(N_{i}^{\text {nonself }}-N_{i}^{\text {self }}\right)+\varepsilon
$$

$\pi_{d}^{\text {nonself }}-\pi_{d}^{\text {self }}$ is the difference in dictator payoffs between the non-selfish and the selfish allocation. $N_{i}^{\text {nonself }}-N_{i}^{\text {self }}$ is the difference in social appropriateness ratings between the two options, computed as explained above. We also estimate a constant $\alpha$ which captures the general tendency to choose non-selfish option.

\footnotetext{
${ }^{7}$ Since we fitted a power curve to both advantageous and disadvantageous allocations, we decided to use the ratings for 30/30 allocation for both curves. Given that we need only one estimate for each allocation, we decided to use the one from the advantageous curve, since the 30/30 split was only paired with other advantageous allocations. We, nonetheless, tested whether estimates from the two curves differed in their predictions using a paired $t$-test, and found no significant difference $(t(165)=-1.54, p>.1)$.
} 


\begin{tabular}{|c|c|c|c|c|c|}
\hline \multirow[b]{2}{*}{ Coefficient } & \multicolumn{3}{|c|}{ Quartiles } & \multirow[b]{2}{*}{ Rank-sum test } & \multirow[b]{2}{*}{$\operatorname{Pr}($ non-selfish $)$} \\
\hline & First & Median & Third & & \\
\hline$\alpha_{i}$ & -4.04 & -1.38 & -0.21 & $p<0.001$ & $-3.026^{* * *}$ \\
\hline$\beta_{i}$ & 0.00 & 0.02 & 0.13 & $p<0.001$ & $0.021^{* * *}$ \\
\hline$\gamma_{i}$ & 0.00 & 1.27 & 3.45 & $p<0.001$ & $1.265^{* * *}$ \\
\hline$N$ observations & & & & & 30,212 \\
\hline$N$ groups/subjects & & & & & 166 \\
\hline
\end{tabular}

Table 1: Summary of the coefficients estimates from individual regressions and a random effects logit regression on all data (errors are clustered by subject). In individual regressions, the participants who only made selfish choices are excluded. ${ }^{* * *}$ stands for $p<0.001$.

Table 1 shows the summary of the estimates of the coefficients across participants. Notice that the distribution of $\alpha_{i}$ lies mostly in the negative domain with zero being in the 4th quartile, and the distributions of $\beta_{i}$ and $\gamma_{i}$ are in the positive domain with zero in the 1st quartile. Ranksum tests show that all three coefficients at sample level are significantly different from zero. The same conclusion can be made from a random effects logit regression that includes all data (last column of Table 1): the coefficients on personal utility, norm utility, and intercept are highly significant and have the predicted signs. We conclude that the choices of the participants are driven by the norm-dependent utility maximization and, thus, by meta-context since the elicited norms used in the estimation conform with those in the standard DG.

As an additional test of consistency of the choices with norm-following, we test our hypothesis that $\gamma_{i}$ represent participants' rule-following propensities and, thus, should be correlated with the estimates obtained in the RF task. Indeed, Spearman's rank correlation between $\gamma_{i}$ and the number of balls put in the blue bucket is $\rho=.29(p<.001)$. We also find that $\gamma_{i}$ correlates with the proportion of non-selfish choices (Spearman's $\rho=.44, p<.001$ ), which provides additional support to the hypothesis that participants choose non-selfish options because they follow norms.

Our analysis up to this point has provided support for the norm-dependent utility specification, with norms coming from meta-context, and has shown that the rule-following propensity can explain the non-selfish choices in mini-DGs. However, the utility function that we estimated is essentially outcome-based since the normative term assigns one value to each allocation based on the meta-context and does not take into account the possible influence of the unchosen option. ${ }^{8}$ Therefore, this analysis does not capture any choice-set dependence that could still be an important factor that drives participants' choices. In the following sections we analyse regression residuals to see how well the norm-dependent utility, specified above, describes the data and check whether choice-set dependent utility is needed to improve the fit.

\footnotetext{
${ }^{8}$ In the logistic regression the two utilities are compared. However, this dependency models the choice process and not the changes in utility due to the presence of another option.
} 


\subsection{Anomalies in Non-Selfish Choices}

In order to understand how well the elicited norms explain behaviour, we look at the norm differences in the subsets of mini-DGs in which the change in personal payoff between the two alternatives is fixed. According to the norm-dependent utility, in such cases only differences in norms should influence the probability of non-selfish choices. Figure $3 \mathrm{~A}$ plots $N_{i}^{\text {nonself }}-$ $N_{i}^{\text {self }}$, the difference in norms between the non-selfish and selfish options, as dependent on the lower payoff in the advantageous mini-DGs with payoff difference equal to $5 .{ }^{9}$ In these trials the model predicts that the more equal the payoffs are in each allocation, the more appropriate it is to choose the non-selfish option. For instance, according to the normative ratings, choosing $30 / 30$ over $35 / 25$ is considered more socially appropriate (average norm difference $\approx 0.38$ ) than choosing $55 / 5$ over $60 / 0$ (average norm difference $\approx 0.18$ ).
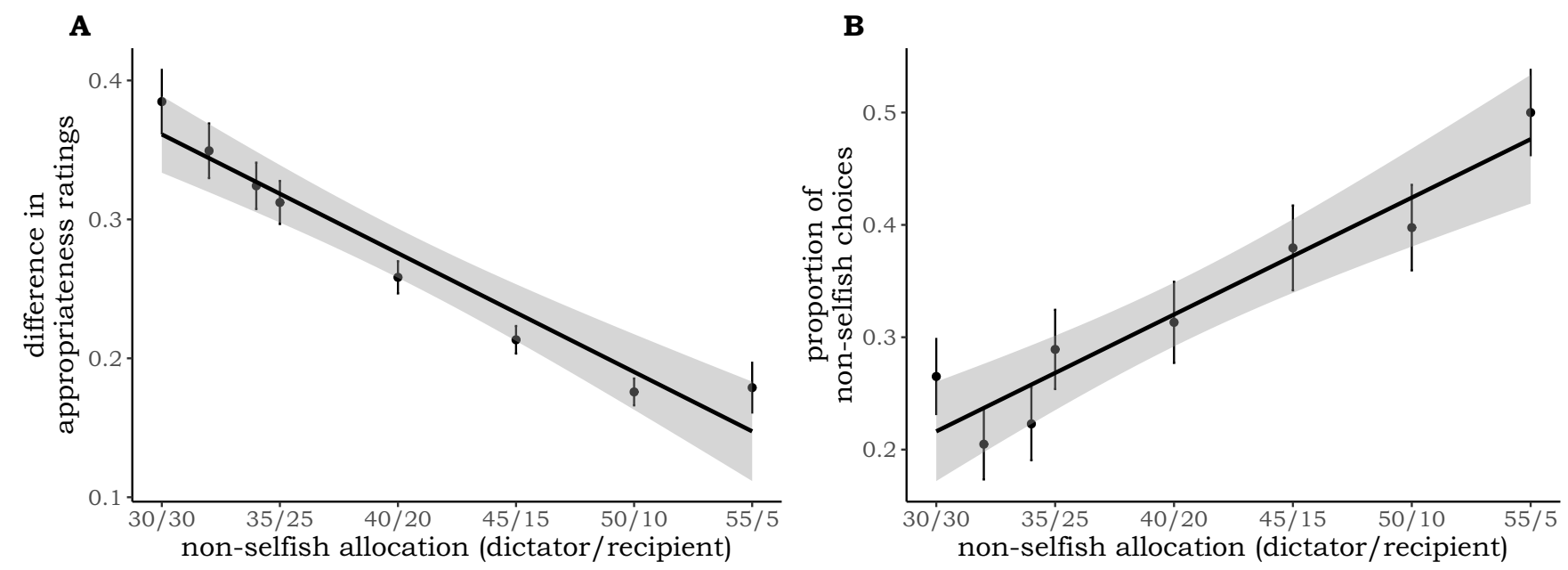

Figure 3: Advantageous mini-DGs with payoff differences equal to 5: A. Norm differences as dependent on the non-selfish dictator's payoff; B. Proportion of non-selfish choices as dependent on the non-selfish dictator's payoff.

If we assume that participants do care about complying with norms-and this is supported by the positive coefficients on the normative term in the regressions-participants should be less selfish in mini-DGs with more equal options as compared to mini-DGs with less equal options. What we observe instead is that participants display the exact opposite behaviour. Figure 3B shows that they are less selfish when alternatives are less equal. The same is true when we analyse the mini-DGs with other fixed payoff differences (Figure 10 in Appendix A). ${ }^{10}$

This discrepancy should also be reflected in the regressions. We check whether the residuals show an anomalous trend (i.e., depend monotonically on payoff when they should not) for

\footnotetext{
${ }^{9}$ We will distinguish between advantageous mini-DGs in which both allocations are advantageous and disadvantageous mini-DGs in which one of the allocations is disadvantageous.

${ }^{10}$ This relationship is not present among the disadvantageous mini-DGs, where meta-context norm predictions match participants' behaviour.
} 
advantageous mini-DGs. ${ }^{11}$ We analyse the residuals using locally weighted scatter-plot smoothing, which plots residuals against the payoffs in the non-selfish option $\left(\pi_{d}^{\text {nonself }}\right.$ and $\left.\pi_{r}^{n o n s e l f}\right)$. As it can be seen in Figure 4, the residuals show a positive trend: a linear regression confirms that the tendency is significant (residuals as dependent on non-selfish payoff, $p<.001$, see Column 1 in Table 6, Appendix A for the regression). ${ }^{12}$

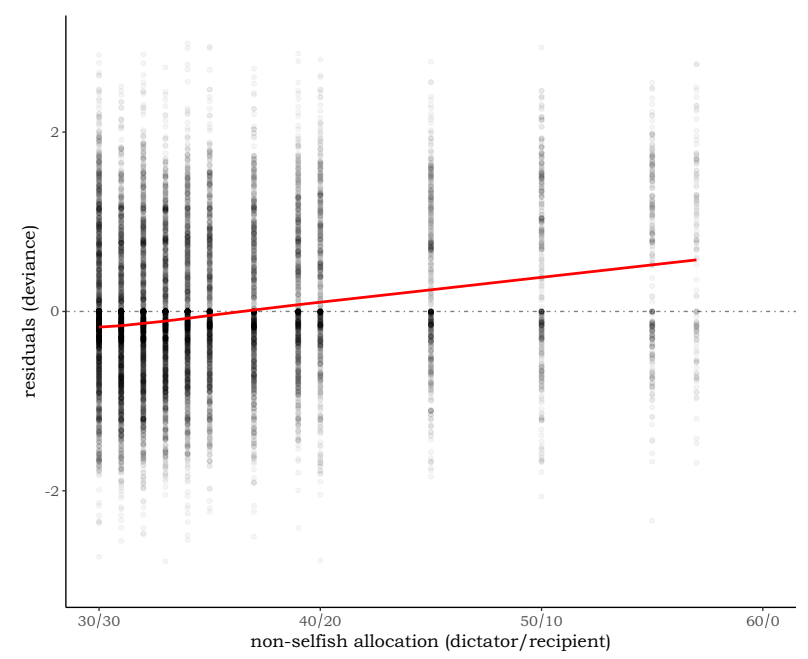

Figure 4: Locally weighted scatter-plot smoothing of deviance residuals across all individual regressions.

There are two reasons why we may observe the trend in residuals. One is the misspecification of the norm-dependent utility. It is possible that the shape of the norm function that we get from the data is influenced by the design and does not reflect correctly the perceived appropriateness of allocations. For example, in Figure 3 the increase in the proportion of non-selfish choices would be qualitatively consistent with the change in differences of appropriateness ratings if the norm function were concave instead of convex. The second reason is that the misspecification could originate from the choice-set dependence that outcome-based utility functions are not able to capture. In what follows we examine the first possibility (misspecified norm function) by fitting other outcome-based utility functions to see if the trend in the residuals disappears. Specifically, we test a polynomial utility and a concave norm utility.

The regression with polynomial utility tests the idea that linear norm-dependent utility has not enough degrees of freedom to explain participants' behaviour. We allow personal payoffs and the norm function to interact in a non-linear fashion. We test a set of such models which contain polynomial terms up to degree 4. According to the Bayes Information Criterion (BIC), the best model is that of degree 3 (BIC: 18153), which shows that the increase in degrees of freedom does not improve the fit of the utility after some point. Figure 12 in Appendix A shows the

\footnotetext{
${ }^{11}$ We drop participants who never chose a non-selfish option from this analysis because they are unaffected by any characteristics of the task.

${ }^{12} \mathrm{We}$ also check the residuals for disadvantageous mini-DGs but do not find any significant anomaly for the current nor for the other regressions (see below). A plot for the disadvantageous mini-DGs residuals is presented in Appendix A, Figure 11. See Column 7 in Table 6, Appendix A, for the regression.
} 
residuals of the winning model: the trend is still statistically significant ( $p<.001$, see Column 2 in Table 6, Appendix A, for the regression). This analysis demonstrates that the complexity of the model does not help to explain away the trend in residuals.

The regression with the concave norm utility is based on the assumption that the trend in the residuals is the result of a distortion in our measurement of social appropriateness. It might be the case that the procedure we used to elicit appropriateness ratings provides a biased estimate of actual beliefs. For instance, the complete equality allocation (30/30) might be particularly salient to participants, so much that it is reported as being much more appropriate than even a slight deviation from it (e.g., 35/25), which in turn is rated as significantly less appropriate. ${ }^{13}$ As a result, the norm function is 'forced' to be convex, while it may actually be the case that participants do not really consider the allocations around equal split as being much normatively distant from it. Along the same lines, participants assign almost the same ratings to giving little (e.g., 55/5) and giving nothing (e.g., 60/0) to the recipient, whereas in reality these two options may be perceived as very different. Such bias, created by the norm-elicitation task, can, in principle, lead to the utility misspecification. To test whether we can eliminate the trend in the residuals, we replace the norm function estimates with a fixed concave norm function.

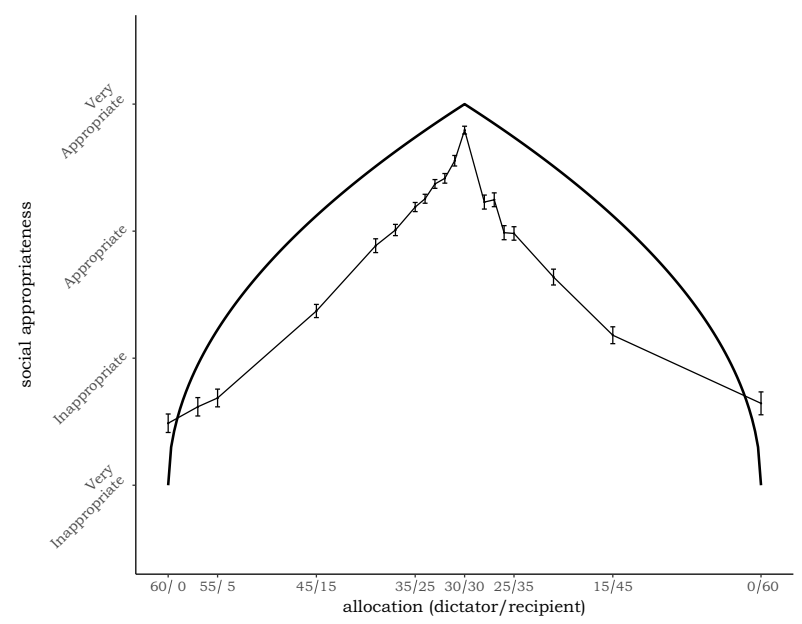

Figure 5: Concave norm function overlapping the data from norm-elicitation task.

We model the concave norm as a power function and fix the three sets of parameters: $a=-1$, $b=2, c \in\{0.1,0.5,0.8\}$. Figure 5 illustrates the concave norm function with $c=0.5$. Unlike in our data, here the decrease in appropriateness is less steep around the 30/30 allocation and more steep around very unequal allocations $(60 / 0,0 / 60)$. For simplicity, the same values are used for both advantageous and disadvantageous allocations. For all three values of $c$ the trend in residuals remains (for $c=0.5$ Figure 13 in Appendix A shows the plot). The coefficients from the linear regressions of residuals on non-selfish payoffs are significantly different from zero for all three models ( $p<.005$, see Columns 3-5 in Table 6, Appendix A, for the regressions).

\footnotetext{
${ }^{13}$ This might be the case because the norm elicitation task is essentially a coordination game in which allocation $30 / 30$ is focal (Sugden, 1995).
} 
Interestingly, the extremely concave norm function with $c=0.1$ does explain well the choices between very unequal allocations, but misses out on the ones close to the equal split, while the least concave function with $c=0.8$ does the opposite.

Overall, this analysis shows that the outcome-based utilities that we considered are not very good at explaining the observed behaviour.

\subsection{Choice-Set Dependence}

In the previous section we tried to correct for the trend in residuals with two outcome-based utility specifications. However the problem persisted. Therefore, we try a new approach that takes into account the possible dependence of the utility of each option on the alternative, in other words, choice-set dependence. We hypothesise that the decision to choose a non-selfish option is modulated by its relative cost, which depends on how much the dictator loses (in percent) and how much the recipient gains (in percent) if the non-selfish option is chosen. Note that relative cost violates an important tenet of the outcome-based utility models where it is assumed that decision makers first attribute a value independently to each alternative and then compare these values to select the optimal option. Relative cost, instead, implies that the value of each allocation depends on the other available alternative.

To represent relative cost, we first notice that the dictator's direct cost of choosing the nonselfish option is $d=\pi_{d}^{\text {self }}-\pi_{d}^{\text {nonself }}>0$, which is also the gain of the recipient $d=\pi_{r}^{\text {nonself }}-$ $\pi_{r}^{\text {self }}$, since the sum of payoffs for each option is constant. We define the percent losses of the dictator and gains of the recipient as

$$
C_{d}=\frac{d}{\pi_{d}^{\text {self }}} \text { and } C_{r}=\frac{d}{\pi_{r}^{\text {nonself }}}
$$

For the dictator, $C_{d}$ is the loss from choosing the non-selfish option relative to the highest payoff. For the recipient, $C_{r}$ is the gain measured relatively to the recipient's highest payoff (when the non-selfish option is chosen by the dictator). We hypothesise that dictator's decision depends on $C_{r}-C_{d}$. For example, for the choice between $60 / 0$ and $55 / 5$, by choosing the non-selfish option the dictator loses only around $8 \%$ of his maximal payoff, while the recipient gains $100 \%$. In such situation the dictator might be willing to be more generous than when the difference in percentages is smaller. We are not the first to notice that relative cost might play a role in dictator games. For example, (Smeets et al., 2015) analyse sharing decisions by millionaires and find that they are extremely generous when they are paired with a low-income recipient: around half of them give the entire $€ 100$ that they are endowed with. This effect might also manifest itself as maximin preferences, when participants sacrifice payoff efficiency for the sake of higher payoff for the 'poorest' participant (Engelmann and Strobel, 2004; Baader and Vostroknutov, 2017). 
We estimate the following regression:

$$
\operatorname{Pr}(\text { non-selfish choice })=\alpha_{i}+\beta_{i} \cdot\left(\pi_{d}^{\text {nonself }}-\pi_{d}^{\text {self }}\right)+\gamma_{i} \cdot\left(N_{i}^{\text {nonself }}-N_{i}^{\text {self }}\right)+\delta_{i} \cdot\left(C_{r}-C_{d}\right)+\varepsilon \text {. }
$$

The results are presented in Table 2 . We see that the distributions of $\alpha_{i}, \beta_{i}$, and $\gamma_{i}$ are very similar to those from the individual regressions without relative cost in Table 1. The same holds for the logit regression on all data shown in the last column. The individual values of $\delta_{i}$ are mostly positive, which is supported by the rank-sum test and the significance of the coefficient in the alldata logit regression. Thus, the probability of non-selfish choice increases when $C_{r}-C_{d}$ is large or, in other words, when both allocations in a mini-DG give most of the payoff to the dictator.

\begin{tabular}{rrrrrrr}
\hline \multicolumn{5}{c}{ Quartiles } & & \\
\cline { 2 - 5 } & First & Median & Third & Rank-sum test & $\operatorname{Pr}$ (non-selfish) \\
\hline$\alpha_{i}$ & -5.06 & -1.91 & -0.45 & $p<0.001$ & $-3.509^{* * *}$ \\
$\beta_{i}$ & 0.00 & 0.04 & 0.16 & $p<0.001$ & $0.034^{* * *}$ \\
$\gamma_{i}$ & -0.00 & 0.66 & 3.14 & $p<0.001$ & $0.609^{* * *}$ \\
$\delta_{i}$ & 0.00 & 2.69 & 6.50 & $p<0.001$ & $4.073^{* * *}$ \\
\hline$N$ groups/subjects & & & & & 30,212 \\
\hline
\end{tabular}

Table 2: Summary of the coefficients estimates from individual regressions and a random effects logit regression on all data (errors are clustered by subject). In individual regressions, the participants who only made selfish choices are excluded. ${ }^{* * *}$ stands for $p<0.001$.

Introducing the relative cost term to the regression eliminates the trend in residuals $(p=.97$, linear regression in Column 6, Table 6 Appendix A), which suggests that the model successfully captures the features of participants' behaviour (Figure 6). Even though some of participants' choices are explained by the normative term in the utility, there is a significant portion of variance not captured by it, but which is explained by the context-dependent term.

As a control measure, we check whether introducing the relative cost term affects the correlation of the norm coefficients $\gamma_{i}$ with the rule-following propensity and the proportion of non-selfish choices. The correlation with the number of balls in the blue bucket is weakened, but still significant (Spearman's $\rho=.17, p=.031$ ), as is the correlation with the proportion of non-selfish choices (Spearman's $\rho=.17, p<.033$ ).

These results demonstrate that the choices in mini-DGs are significantly affected by the context. In particular, the dictators become more generous when their 'wealth' is much higher than that of the recipients. 


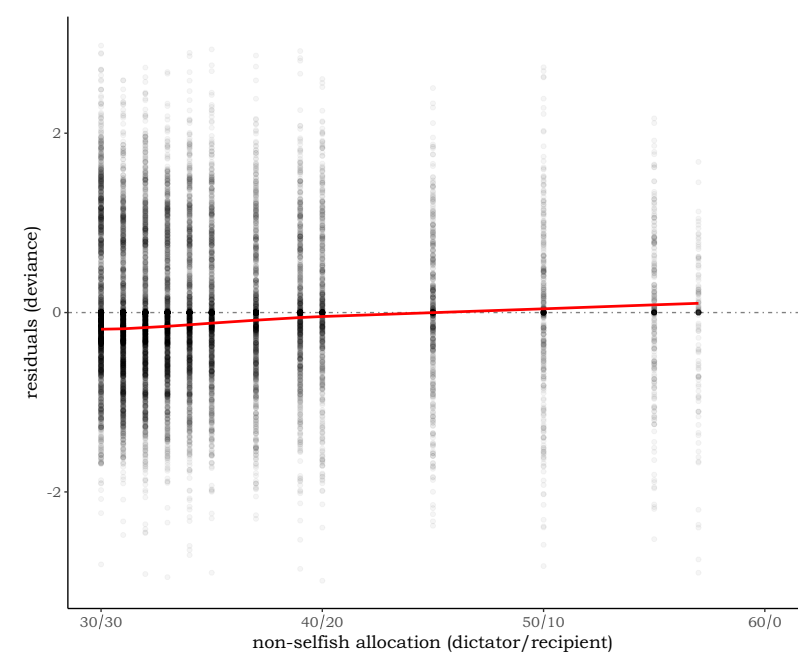

Figure 6: Locally weighted scatter-plot smoothing of deviance residuals across all individual regressions with relative cost.

\subsection{The Nature of Choice-Set Dependence}

The results of the previous section show the presence of the choice-set effect on participants' generosity. However, it remains unclear whether this effect is part of norms-driven behaviour or is a separate phenomenon. More precisely, there are two possibilities: 1) the choice-set effect is working independently from norms, which are correctly represented by the ratings obtained in the norm-elicitation task; 2) the choice-set effect is related to norms but is not captured by the normative ratings. The latter possibility might, in turn, stem from two sources. It can be that our norm-elicitation task in not flexible enough to capture the choice-set effect or that participants, when evaluating the appropriateness of different allocations, do not consciously perceive it. Unfortunately, with our design we cannot tell these two apart (see Section 4 for discussion); however, we can definitely test whether choice-set effect is related to norm-following or not.

Our previous analysis has shown that the rule-following propensity, estimated in the RF task, is a good proxy for the coefficient $\gamma$ in the norm-dependent utility function: it correlates with the proportion of non-selfish choices and with the individual $\gamma_{i}$ coefficients obtained from the normdependent utility regressions. Thus, in order to test whether choice-set effect is norm-related or not, we can use these estimates to see if there is a difference in the strength of the effect between rule-followers and rule-breakers.

Figure 7 shows the data presented in Figure 3 separately for rule-breakers and rule-followers, the two groups of participants defined by the median split of the number of balls in the blue bucket in the RF task. We see that the normative differences are the same for both groups: this is not surprising given that normative ratings, as we have seen, are not statistically different between the two groups. However, rule-followers are more generous than rule-breakers overall and show stronger reaction to the relative $\operatorname{cost} C_{r}-C_{d}$, which increases in dictator's payoff (the slope for rule-followers is higher). This suggests that rule-followers are more influenced 

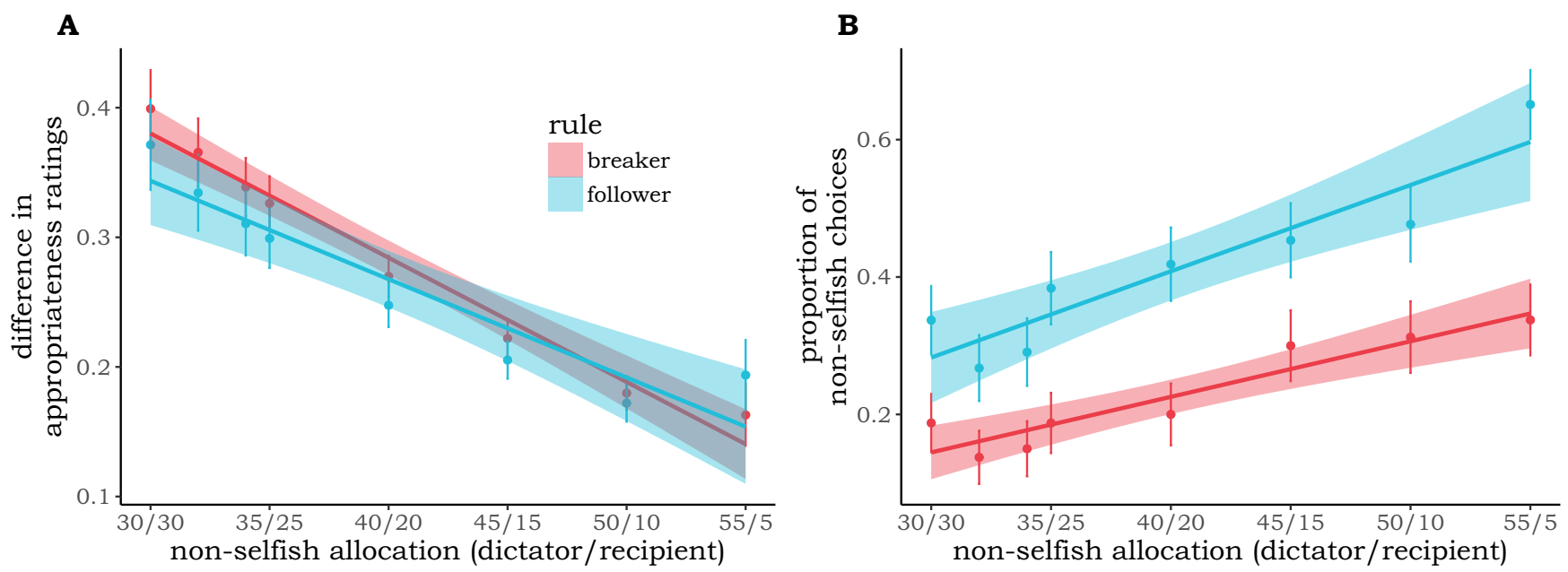

Figure 7: Advantageous mini-DGs with payoff differences equal to 5: A. Norm differences as dependent on the non-selfish dictator's payoff; B. Proportion of non-selfish choices as dependent on the non-selfish dictator's payoff.

by the choice set than rule-breakers, which supports the hypothesis that choice-set dependence is norm-related. This finding is also supported by the correlation between the rule-following propensity and the coefficients $\delta_{i}$ from the individual regressions with relative costs discussed in the previous section (Spearman's $\rho=0.20, p=0.009$ ). Positive correlation means that the more rule-following a participant is, the more she reacts to the relative cost by increasing the probability of choosing the non-selfish allocation.

\begin{tabular}{lcc}
\hline & residuals & $\mathrm{SE}$ \\
\hline intercept & $-0.630^{* * *}$ & $(0.056)$ \\
follower & $-0.285^{* * *}$ & $(0.080)$ \\
$\pi_{d}^{\text {nonself }}$ & $0.018^{* * *}$ & $(0.002)$ \\
follower $\times \pi_{d}^{\text {nonself }}$ & $0.011^{* * *}$ & $(0.002)$ \\
\hline$N$ observations & 30,212 & \\
$N$ groups/subjects & 166 & \\
\hline
\end{tabular}

Table 3: OLS regression of the residuals from the logit regression in Table $1 .{ }^{* * *}$ stands for $p<0.001$.

To support these results even further, we take the residuals (deviance) from the random effects logit regression reported in Table 1 and regress them on the non-selfish payoffs of the dictator, the dummy for rule-followers, and their interaction. Table 3 shows that the interaction term is significant and large, in comparison with the coefficient on the payoff. Thus, the trend in residuals is stronger for rule-followers, which means that the choice-set effect is more pronounced for them. Figure 14 in Appendix A shows this result graphically. 
We can conclude that the choice-set effect that we observe in our data is definitely related to the rule-following propensity, and, thus, to norm-following in general, even though our normelicitation task does not capture it. In the next section we discuss the possible implications of this finding.

\section{Discussion}

Summary of the results. The goal of the research project reported in this paper was to study the influence of meta-context and choice set on pro-social behaviour. Our original intuition was that by measuring the normative perceptions of actions in different mini-Dictator games we will be able to observe the influence of the choice-set context and, consequently, explain the contextdependent behaviour. However, what we uncovered is that the normative ratings of allocations in mini-DGs, instead of being linked to the specific choice set of each game, reflect meta-context, which derives from a superset of hypothetical alternatives (a standard DG). The ease and unanimity of participants in expressing the normative values as coming from a meta-context suggest that it is natural for them to think in such terms. More importantly, we demonstrate that these meta-context considerations do explain a substantial portion of variance in the choices and, therefore, should be taken into account in normative models of social behaviour (e.g., Kimbrough and Vostroknutov, 2018a).

This being said, we also find that in some circumstances the observed choices do not conform with the meta-context norms that our participants agree on. In particular, when the payoffs of a dictator are much higher than those of a recipient in both allocations of a mini-DG, there is a discrepancy between the normative evaluations and the proportion of generous choices: participants behave much more pro-socially than what meta-context predicts. We demonstrate that this bias can be explained by assuming that there is an influence of choice set on participants' decisions, which is expressed as a relative cost of being selfish. To strengthen this result, we show that several outcome-based utility specifications, which have many degrees of freedom but do not consider the choice-set effects, fail to explain this behaviour. Finally, we find strong association between this choice-set effect and the individual propensity to follow rules, which suggests that the influence of the choice set is normative in nature.

Overall, we found strong support for our original hypothesis that choice-set dependence in mini-DGs is related to normative considerations. However, the situation is more complex than we thought. There are two normative components that shape pro-social behaviour. One, derived from meta-context, represents an 'absolute' normative value of each allocation, irrespective of 
other available options. Another, based on choice set, is a 'relative' normative value determined by the comparison of an allocation with the alternatives available in the specific situation.

Meta-context and social preferences. The idea that some social decisions are explained by the presence of an absolute normative value, which is a function of an outcome and not of the choice context, may sound similar to certain definitions of social preferences. However, we would like to point out that this resemblance is superficial. The meta-context of a mini-DG that we talk about comprises all hypothetically conceivable allocations of a specific type. Since the participants only choose among divisions of 60 tokens, the meta-context is a standard Dictator game with possible allocations $\left\{\left(\pi_{d}, \pi_{r}\right) \mid \pi_{d}, \pi_{r} \geq 0\right.$ and $\left.\pi_{d}+\pi_{r}=60\right\}$. Notice that this would be different if participants were sometimes also presented with an option to take money from the other player. In this case, meta-context would include additional allocations where dictator can earn more than 60 tokens. As is well-known from the studies by List (2007), Bardsley (2008), and Cappelen et al. (2013), in such circumstances the choices become much less generous than in the standard DG setting, and this is exactly what we would expect if participants were presented with taking options. ${ }^{14}$ Thus, meta-context represents all possible allocations similar to those experienced in the experiment and not all allocations that can happen in principle. In this sense, it is still a context that does not apply in all situations. Following this mode of thinking, the immutable universal social preferences may be seen as coming from some grand meta-context that covers all possible kinds of social interactions. It may well be that this is indeed the case, and that such considerations do play a role in social decisions. However, given their universality, it is very plausible that they will be overshadowed by more specific contextual effects.

Norm elicitations and choice-set dependence. As we mentioned in Section 3.6, there is an unclarified issue related to the data obtained from the norm-elicitation task. Why is it that participants, when asked to guess the prevalent social norms in mini-DGs, express normative values for each allocation as if they were coming from a meta-context? Why do not they combine the absolute and relative normative components in their evaluations? Unfortunately, our design does not allow us to answer these questions directly. Nevertheless, we can discuss the possible reasons behind this phenomenon.

The simplest possibility is that the norm-elicitation procedure itself precludes participants from correctly expressing their normative views. The fact that the 19 mini-DGs used for norm elicitation are presented one after another might be conducive to giving the same rating to an allocation, regardless of the alternative option. This effect might be exacerbated by the coordi-

\footnotetext{
${ }^{14}$ Similar line of reasoning about changing meta-context can be applied to an ongoing debate regarding the availability of punishment options in experiments. Some researchers claim that the ubiquity of punishment observed in, say, repeated Public Goods games is due to an experimenter demand effect created by the fact that only punishment is available. According to this argument, rewards should also be possible in order to eliminate the demand effect. In our opinion it is not that simple. The introduction of rewards would change the nature of the meta-context, which can lead to completely different social attitudes than in case when only punishment is available.
} 
nation game structure of the task: it might be tempting to think that everyone in a session will give the same rating to each allocation to simplify the coordination problem.

On a deeper level, this problem might have nothing to do with the norm-elicitation task per se, but rather with how the normative evaluation is performed by the brain. It is possible that people, when asked to give appropriateness ratings, process information differently from the situations when they actually have to make normative decisions. In the former case, they might tend to provide general judgements related to the meta-context of a given situation without thinking about specifics, while in the latter they start taking choice set into account since, after all, they must make a choice. This distinction may be similar to attentional mechanisms responsible for the visual exploration, which operates on two levels: a general first impression is quickly constructed, and then, if necessary (e.g., when a choice should be made), a more detailed analysis is performed in order to elaborate on the first impression (Duchowski, 2007). We hope that future experiments will clarify the situation around the normative perceptions of decisions affected by a choice-set context.

Theoretical and experimental implications. Finally, we discuss some implications of our study for theoretical modelling and design of experiments. If, as we suspect, the influence of metacontext and the choice set spreads beyond mini-DGs, then it becomes important to appropriately model their effects.

From the theoretical perspective, it would be interesting to understand what exactly constitutes a meta-context for each given environment. Another natural application is in dynamic games: from the perspective of a player who acts in a subgame, the outcomes of the entire game can be viewed as meta-context (see Kimbrough and Vostroknutov, 2018a). Experimentally, sensitivity to meta-context implies that care should be taken in within-subject designs where participants are presented with a series of similar tasks. In such cases the knowledge of the past possibilities can affect how the actions in a game at hand are viewed normatively (e.g., Chlaß and Moffatt, 2012).

The main challenge for choice-set dependence is to develop a method of incorporating the alternatives into the utility function. Kimbrough and Vostroknutov (2018a) propose one such theoretical approach. They consider the same norm-dependent utility specification that we used in this study, but calculate the norm associated with each allocation as dependent on all other allocations that can be realised in a game. Thus, the utility of each allocation stops being solely outcome-based, as now it depends on the alternatives through the norm function. It should be mentioned that in their model meta-context can also be easily accounted for, though the authors do not suggest how it should be chosen.

Another important line of future investigation is norm-elicitation tasks. As we mentioned earlier, it remains unclear whether the original task proposed by Krupka and Weber (2013) creates some biases in elicited norms or not. For example, it might be the case that the convexity of 
the norm functions in DG is an artefact of the elicitation method (see Section 3.2), or that seeming context-independence of the elicited norms is a consequence of participants' attempts at coordination. We are currently working on a new norm-elicitation task that will rectify these and other potential issues.

\section{Conclusion}

After three decades of experiments with the Dictator game (DG) it may seem that the motives of experimental subjects are pretty well understood. Nevertheless, we uncover two effects that, to our knowledge, were not studied together in the previous literature. In our experiment each participant plays 182 mini-Dictator games with two actions and fixed payoff efficiency. After that we ask participants to express their normative evaluations of the actions in 19 of these miniDGs.

The first effect that we discover is what we call meta-context. Specifically, the probability of the non-selfish choice in a mini-DG is modulated by the normative reasoning related to the supergame that contains it, in our case, a standard DG. This means that participants take into account the appropriateness of the available actions as if these actions were part of the standard DG. We conclude this from the observation that the normative ratings, which participants express in the norm-elicitation task, do not depend on the choice set and conform with similar elicitations obtained in a standard DG. Moreover, these normative evaluations explain a sizeable portion of variance in choices.

The second effect is choice-set dependence. While meta-context accounts for some pro-social choices, we find a systematic deviation from its predictions in mini-DGs that give very high payoff to the dictator in both available allocations. In such games participants behave more pro-socially than they would if only meta-context mattered. We show that this is explained by assuming that the utility of each choice depends on the alternative. This dependency can be expressed as a relative cost of non-selfish choice. In particular, participants seem to take into account how important the difference in payoffs between the two allocations is for them and for the recipient in terms of their respective wealths.

Our final finding is that the choice-set effect is not independent from the propensity to follow rules. Thus, the two effects that we observe represent two distinct components of the normative value that participants take into account. This demonstrates that pro-sociality is a complex phenomenon that in each particular case depends on the specifics of the situation and a general context to which this situation belongs. 


\section{References}

BAADER, M. and VOSTROKNUTOV, A. (2017). Interaction of reasoning ability and distributional preferences in a social dilemma. Journal of Economic Behavior E Organization, 142, 79-91.

BARDSLEY, N. (2008). Dictator game giving: altruism or artefact? Experimental Economics, 11 (2), 122-133.

Bolton, G. E. and OCKenfels, A. (2000). Erc: A theory of equity, reciprocity, and competition. American economic review, 90 (1), 166-193.

Cappelen, A. W., Hole, A. D., Sørensen, E. Ø. and Tungodden, B. (2007). The pluralism of fairness ideals: An experimental approach. American Economic Review, 97 (3), 818-827.

-, Nielsen, U. H., Sørensen, E. Ø., Tungodden, B. and Tyran, J.-R. (2013). Give and take in dictator games. Economics Letters, 118 (2), 280-283.

CHANG, D., CHEN, R. and KRUPKA, E. (2018). Rhetoric matters: A social norms explanation for the anomaly of framing. Games and Economic Behavior.

Chlass, N. and Moffatt, P. G. (2012). Giving in dictator games: Experimenter demand effect or preference over the rules of the game?, no. 2012, 044. Jena Economic Research Papers.

Cox, J. C., List, J. A., Price, M., SAdiraj, V. and SAMEK, A. (2016). Moral costs and rational choice: Theory and experimental evidence.

DuchowsKi, A. T. (2007). Eye Tracking Methodology: Theory and Practice. Springer.

ENGELMANN, D. and STROBEL, M. (2004). Inequality aversion, efficiency, and maximin preferences in simple distribution experiments. American economic review, 94 (4), 857-869.

FEHR, E. and SCHMIDT, K. M. (1999). A theory of fairness, competition, and cooperation. The quarterly journal of economics, 114 (3), 817-868.

FISCHBACHER, U. (2007). z-tree: Zurich toolbox for ready-made economic experiments. Experimental economics, 10 (2), 171-178.

Franzen, A. and Pointner, S. (2012). Anonymity in the dictator game revisited. Journal of Economic Behavior E Organization, 81 (1), 74-81.

Hoffman, E., MCCABE, K. and SMITH, V. L. (1996). Social distance and other-regarding behavior in dictator games. The American economic review, 86 (3), 653-660.

Hutcherson, C. A., Bushong, B. and RAngel, A. (2015). A neurocomputational model of altruistic choice and its implications. Neuron, 87 (2), 451-462.

Kahneman, D., Knetsch, J. L. and Thaler, R. H. (1986). Fairness and the assumptions of economics. Journal of business, pp. S285-S300.

KesSler, J. B. and LeIDER, S. (2012). Norms and contracting. Management Science, 58 (1), 62-77.

Kimbrough, E. and Vostroknutov, A. (2016). Norms make preferences social. Journal of European Economic Association, 14 (3), 608-638. 
— and - (2018a). A normative model of social behavior, mimeo, Chapman University and University of Trento.

— and - (2018b). A portable method of eliciting respect for social norms. Economics Letters, 168, $147-150$.

Kimbrough, E. O., Miller, J. B. and Vostroknutov, A. (2018). Norms, frames, and prosocial behavior in games, mimeo, Chapman University, Bocconi University, University of Trento.

KRUPKA, E. and WEBER, R. A. (2009). The focusing and informational effects of norms on prosocial behavior. Journal of Economic psychology, 30 (3), 307-320.

KRUPKA, E. L. and WEBER, R. A. (2013). Identifying social norms using coordination games: why does dictator game sharing vary? Journal of European Economic Association, 11 (3), 495524.

Kuss, K., Falk, A., Trautner, P., Montag, C., Weber, B. and Fliessbach, K. (2015). Neuronal correlates of social decision making are influenced by social value orientation - an $\mathrm{fMRI}$ study. Frontiers in Behavioral Neuroscience, 9.

Liberman, V., SAmuels, S. M. and Ross, L. (2004). The name of the game: Predictive power of reputations versus situational labels in determining prisoner's dilemma game moves. Personality and social psychology bulletin, 30 (9), 1175-1185.

Lightner, A. D., Barclay, P. and Hagen, E. H. (2017). Radical framing effects in the ultimatum game: the impact of explicit culturally transmitted frames on economic decision-making. Royal Society Open Science, 4 (12), 170543.

LIST, J. A. (2007). On the interpretation of giving in dictator games. Journal of Political economy, 115 (3), 482-493.

LÓPEZ-PÉREZ, R. (2008). Aversion to norm-breaking: A model. Games and Economic behavior, 64, 237-267.

McFAdDEN, D. L. (1976). Quantal choice analaysis: A survey. In Annals of Economic and Social Measurement, Volume 5, number 4, NBER, pp. 363-390.

Morgenstern, O. and Von Neumann, J. (1953). Theory of games and economic behavior. Princeton university press.

Smeets, P., BAuer, R. and Gneezy, U. (2015). Giving behavior of millionaires. Proceedings of the National Academy of Sciences, 112 (34), 10641-10644.

Sugden, R. (1995). A theory of focal points. The Economic Journal, pp. 533-550.

ThOMsson, K. and Vostroknutov, A. (2017). Small-world conservatives and rigid liberals: Attitudes towards sharing in self-proclaimed left and right. Journal of Economic Behavior and Organization, 135, 181-192. 


\section{Appendix (for online publication)}

\section{A Additional Analyses}

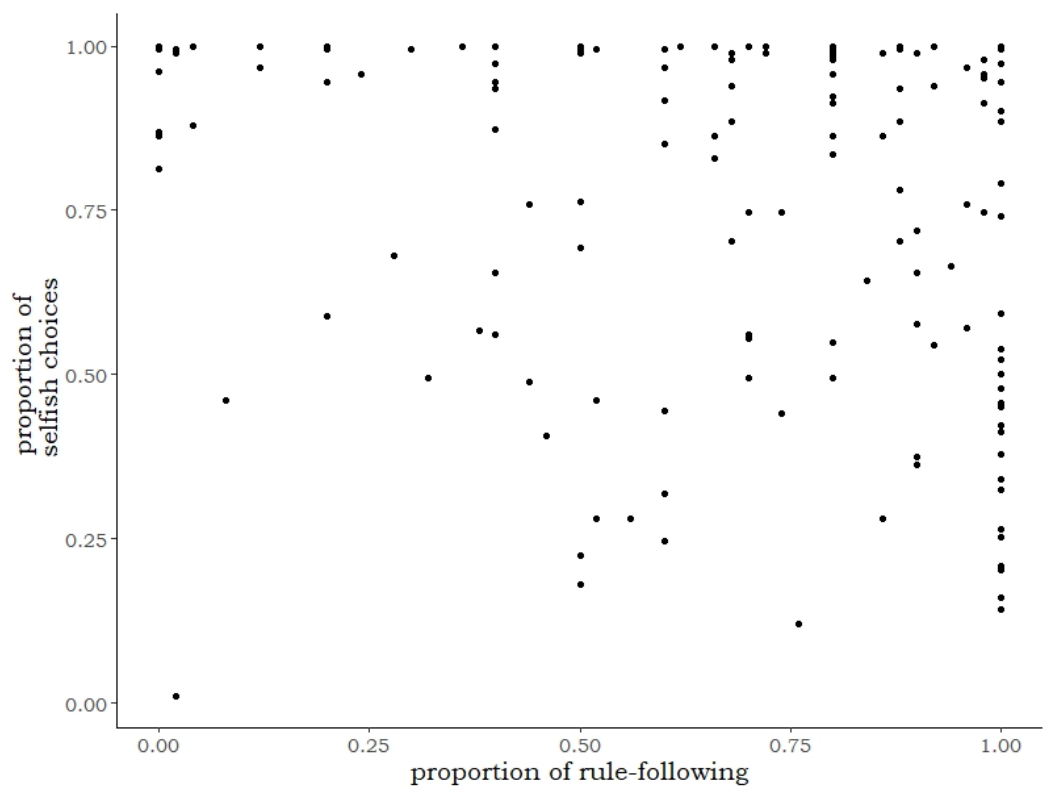

Figure 8: The relation between the rule-following propensity and the proportion of selfish choices. 


\begin{tabular}{|c|c|c|c|c|c|c|c|}
\hline \multicolumn{2}{|c|}{ Allocation } & \multicolumn{3}{|c|}{ A } & \multicolumn{3}{|c|}{ B } \\
\hline A & B & mean & SE & median & mean & SE & median \\
\hline $0 / 60$ & $60 / 0$ & -0.570 & 0.060 & -1.000 & -0.622 & 0.057 & -1.000 \\
\hline $15 / 45$ & $55 / 5$ & -0.213 & 0.044 & -0.333 & -0.546 & 0.050 & -1.000 \\
\hline $21 / 39$ & $57 / 3$ & 0.092 & 0.042 & 0.333 & -0.610 & 0.051 & -1.000 \\
\hline $25 / 35$ & $55 / 5$ & 0.321 & 0.035 & 0.333 & -0.554 & 0.049 & -1.000 \\
\hline $26 / 34$ & $35 / 25$ & 0.325 & 0.037 & 0.333 & 0.482 & 0.026 & 0.333 \\
\hline $27 / 33$ & $33 / 27$ & 0.498 & 0.036 & 0.333 & 0.606 & 0.027 & 0.333 \\
\hline $28 / 32$ & $37 / 23$ & 0.486 & 0.037 & 0.333 & 0.337 & 0.032 & 0.333 \\
\hline $30 / 30$ & $34 / 26$ & 0.847 & 0.028 & 1.000 & 0.502 & 0.028 & 0.333 \\
\hline $30 / 30$ & $39 / 21$ & 0.867 & 0.025 & 1.000 & 0.257 & 0.036 & 0.333 \\
\hline $30 / 30$ & $60 / 0$ & 0.859 & 0.027 & 1.000 & -0.719 & 0.050 & -1.000 \\
\hline $30 / 30$ & $45 / 15$ & 0.880 & 0.024 & 1.000 & -0.096 & 0.039 & -0.333 \\
\hline $31 / 29$ & $37 / 23$ & 0.703 & 0.028 & 1.000 & 0.341 & 0.033 & 0.333 \\
\hline $32 / 28$ & $35 / 25$ & 0.606 & 0.030 & 0.333 & 0.438 & 0.031 & 0.333 \\
\hline $32 / 28$ & $57 / 3$ & 0.614 & 0.032 & 0.333 & -0.566 & 0.055 & -1.000 \\
\hline $33 / 27$ & $34 / 26$ & 0.550 & 0.027 & 0.333 & 0.482 & 0.030 & 0.333 \\
\hline $33 / 27$ & $45 / 15$ & 0.586 & 0.031 & 0.333 & -0.092 & 0.039 & -0.333 \\
\hline $55 / 5$ & $34 / 26$ & -0.526 & 0.051 & -1.000 & 0.526 & 0.030 & 0.333 \\
\hline $60 / 0$ & $45 / 15$ & -0.683 & 0.053 & -1.000 & -0.068 & 0.039 & -0.333 \\
\hline
\end{tabular}

Table 4: Items used in the norm elicitation task and the rating summary. 


\begin{tabular}{rrrrrrr}
\hline & Min. & 1st Qu. & Median & Mean & 3rd Qu. & Max. \\
\hline$a_{a d v}$ & -1.18 & -1.04 & -0.97 & -0.69 & -0.70 & 1.09 \\
$b_{a d v}$ & -1.29 & 1.44 & 1.78 & 1.52 & 1.99 & 2.40 \\
$c_{a d v}$ & 0.00 & 0.98 & 1.49 & 8.53 & 2.14 & 538.38 \\
\hline$a_{d i s}$ & -1.14 & -1.01 & -0.95 & -0.58 & -0.34 & 1.08 \\
$b_{d i s}$ & -0.86 & 1.11 & 1.65 & 1.44 & 1.94 & 2.24 \\
$c_{d i s}$ & 0.00 & 1.35 & 2.29 & 44.16 & 6.83 & 3637.47 \\
\hline
\end{tabular}

Table 5: Summary of the individual parameters for the advantageous and disadvantageous norm functions.

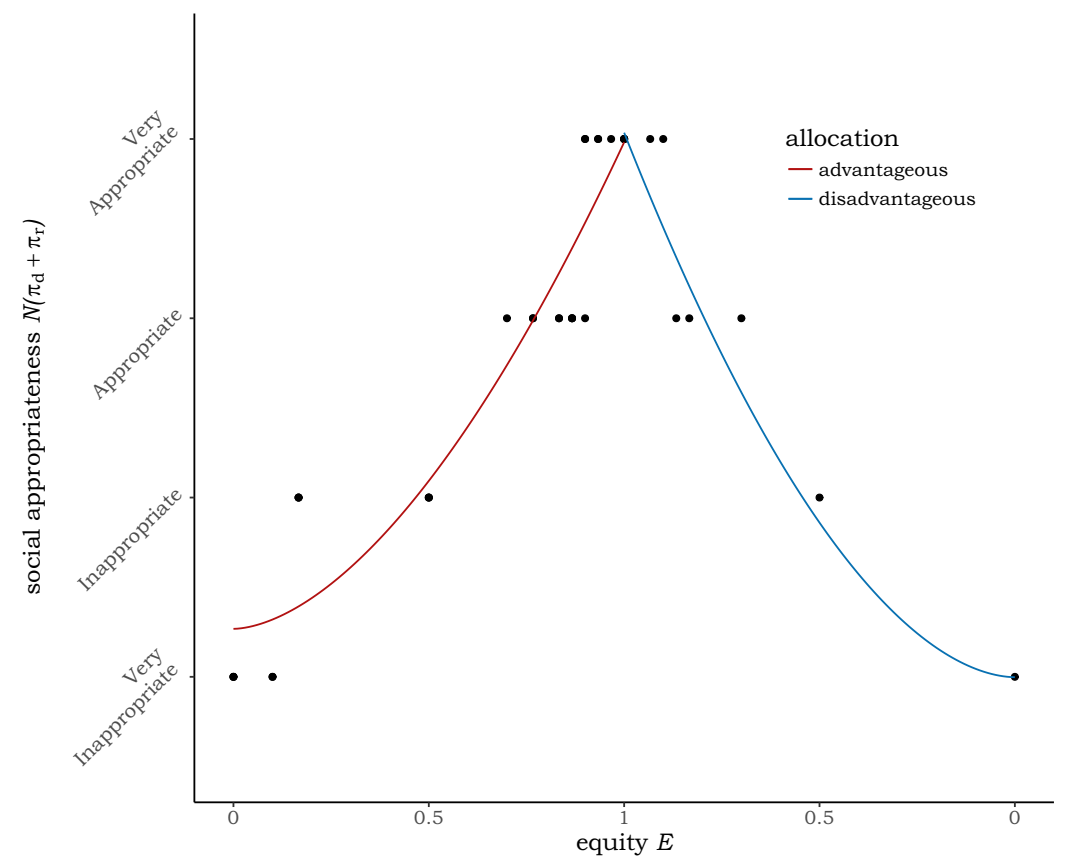

Figure 9: An example of a power function fitting (participant 17). 

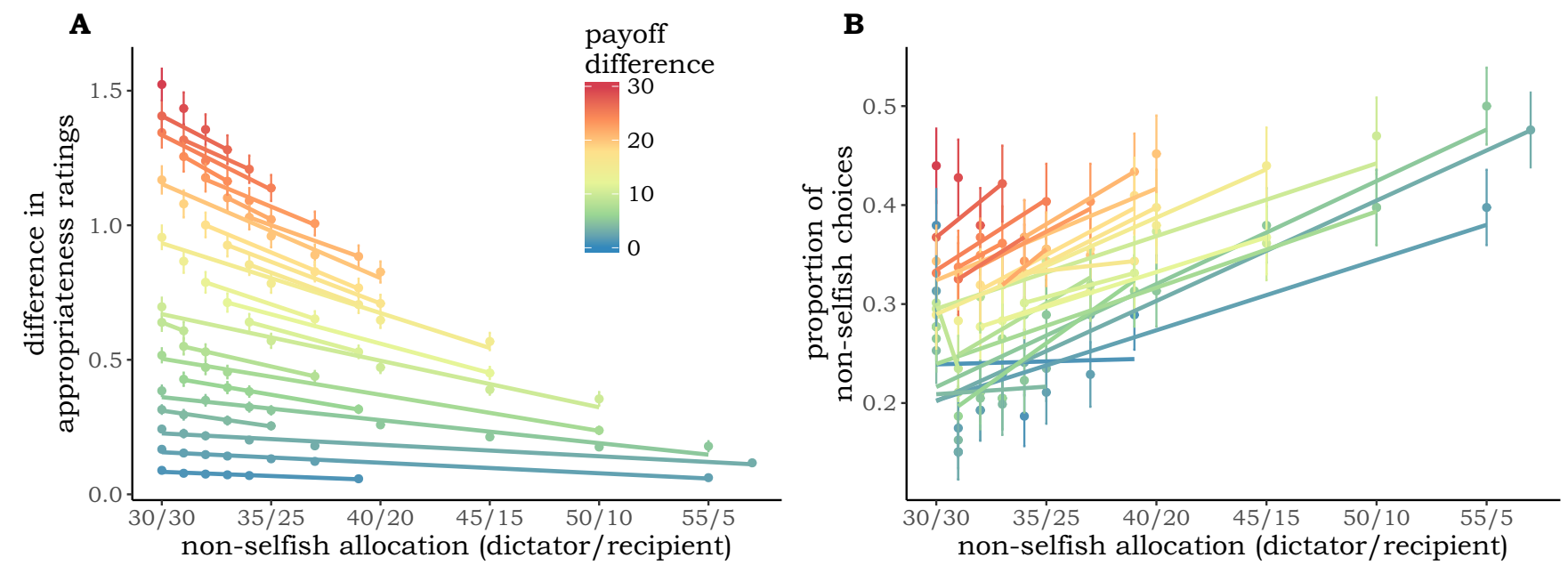

Figure 10: Difference in appropriateness rating and proportion of non-selfish choices for miniDGs with fixed payoff differences (similar to Figure 3).

\begin{tabular}{lccccccc}
\hline & $(1)$ & $(2)$ & $(3)$ & $(4)$ & $(5)$ & $(6)$ & \multicolumn{1}{c}{$(7)$} \\
\hline$\pi_{d}^{\text {nonself }}$ & $0.026^{* * *}$ & $0.013^{* * *}$ & $0.009^{* * *}$ & $0.004^{* *}$ & $0.016^{* * *}$ & 0.000 & 0.000 \\
intercept & $-0.862^{* * *}$ & $-0.401^{* * *}$ & $0.320^{* * *}$ & $-0.119^{* *}$ & $-0.541^{* * *}$ & 0.000 & $-0.125^{* * *}$ \\
\hline$N$ observations & 12,376 & 12,376 & 12,376 & 12,376 & 12,376 & 12,376 & 14,144 \\
\hline
\end{tabular}

Table 6: OLS regression of the residuals from different models. (1) standard norm-dependent utility; (2) degree 3 polynomial utility; (3) concave norm utility $c=0.1$; (4) concave norm utility $c=0.5$; (5) concave norm utility $c=0.8$; (6) standard norm-dependent utility with relative cost; (3) standard norm-dependent utility, disadvantageous allocations; ${ }^{* * *}$, ${ }^{* *}$ stand for $p<0.001$ and $p<0.01$. 


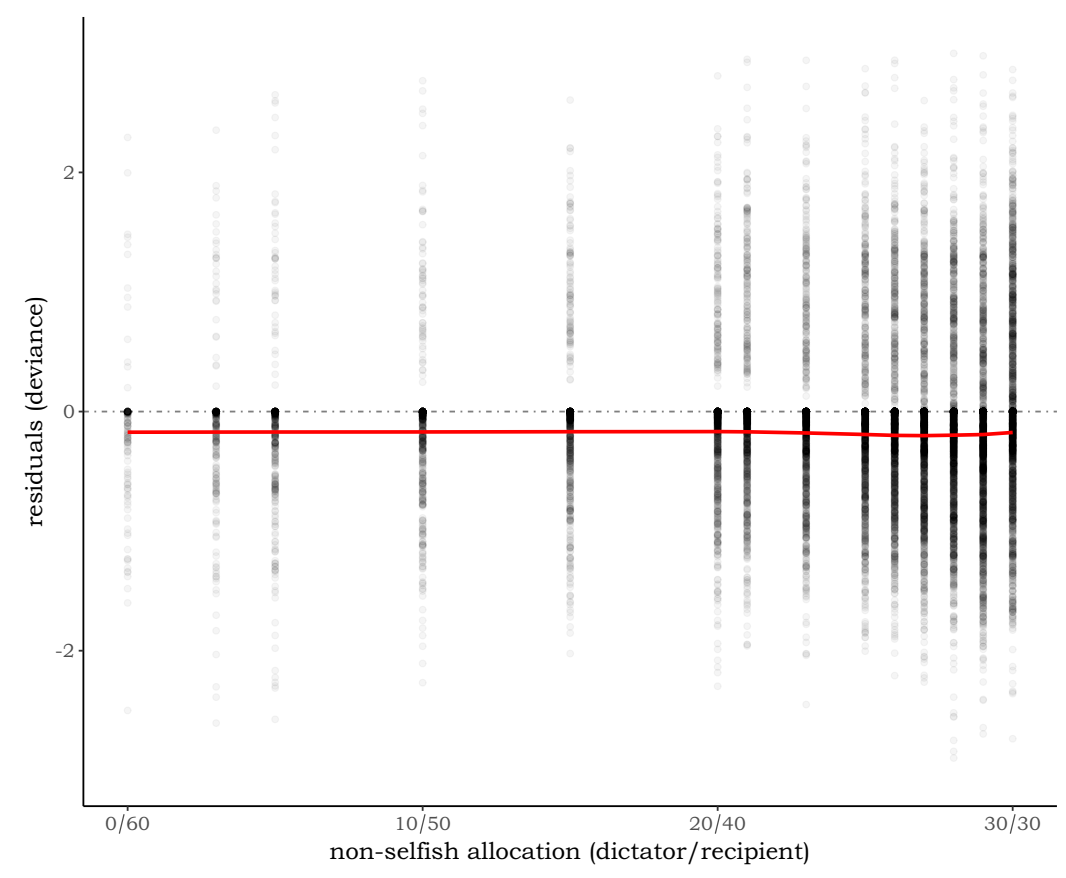

Figure 11: Locally weighted scatter-plot smoothing of deviance residuals for disadvantageous mini-DGs.

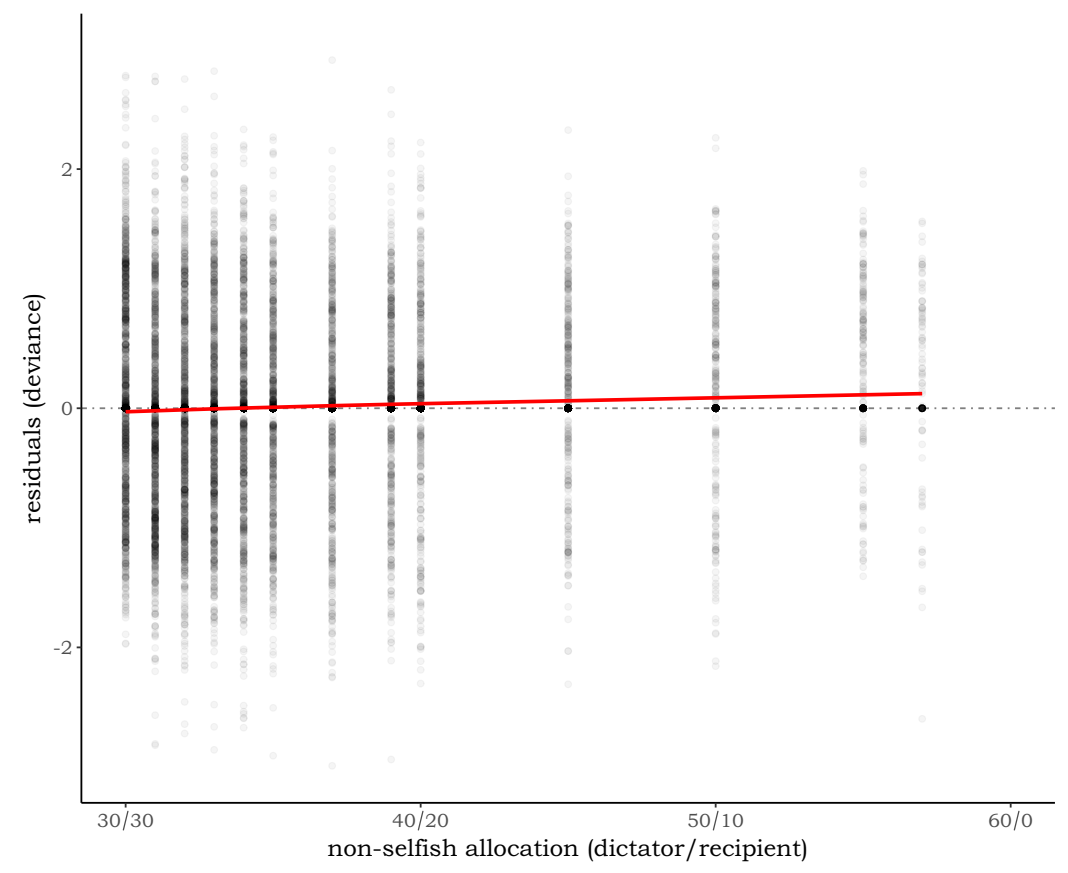

Figure 12: Locally weighted scatter-plot smoothing of deviance residuals across all individual regressions in polynomial regression. 


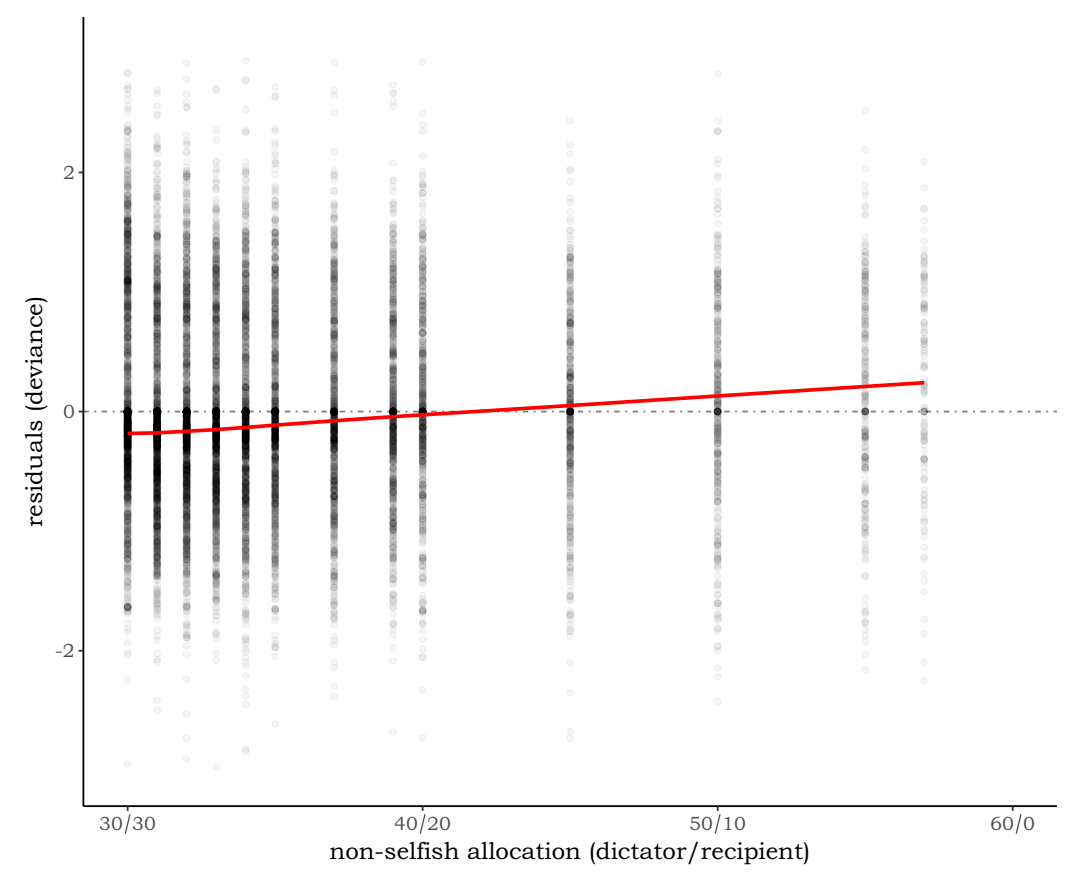

Figure 13: Locally weighted scatter-plot smoothing of deviance residuals across all individual regressions in concave norm regression.

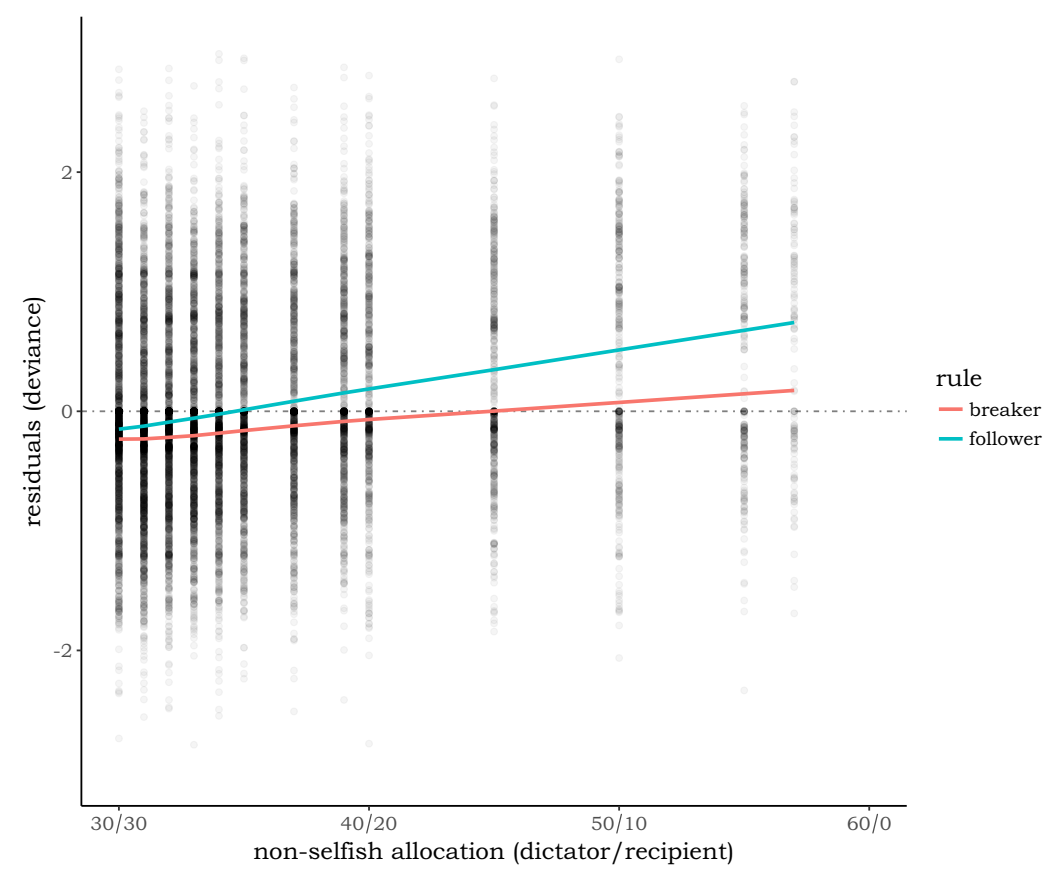

Figure 14: Locally weighted scatter-plot smoothing of deviance residuals across all individual regressions: regressions for rule-followers and rule-breakers. 


\begin{tabular}{|c|c|c|c|c|c|c|c|}
\hline \multicolumn{8}{|c|}{ Allocation } \\
\hline A & B & A & B & A & B & A & B \\
\hline $3 / 57$ & $60 / 0$ & $30 / 30$ & $33 / 27$ & $37 / 23$ & $26 / 34$ & $50 / 10$ & $31 / 29$ \\
\hline $5 / 55$ & $55 / 5$ & $30 / 30$ & $35 / 25$ & $37 / 23$ & $27 / 33$ & $50 / 10$ & $34 / 26$ \\
\hline $5 / 55$ & $60 / 0$ & $30 / 30$ & $39 / 21$ & $37 / 23$ & $30 / 30$ & $50 / 10$ & $35 / 25$ \\
\hline $10 / 50$ & $50 / 10$ & $30 / 30$ & $45 / 15$ & $37 / 23$ & $31 / 29$ & $50 / 10$ & $40 / 20$ \\
\hline $10 / 50$ & $57 / 3$ & $30 / 30$ & $55 / 5$ & $37 / 23$ & $34 / 26$ & $50 / 10$ & $45 / 15$ \\
\hline $15 / 45$ & $50 / 10$ & $30 / 30$ & $60 / 0$ & $37 / 23$ & $35 / 25$ & $50 / 10$ & $57 / 3$ \\
\hline $15 / 45$ & $57 / 3$ & $31 / 29$ & $29 / 31$ & $37 / 23$ & $40 / 20$ & $55 / 5$ & $10 / 50$ \\
\hline $20 / 40$ & $45 / 15$ & $31 / 29$ & $33 / 27$ & $37 / 23$ & $50 / 10$ & $55 / 5$ & $15 / 45$ \\
\hline $20 / 40$ & $55 / 5$ & $31 / 29$ & $35 / 25$ & $37 / 23$ & $57 / 3$ & $55 / 5$ & $23 / 37$ \\
\hline $20 / 40$ & $60 / 0$ & $31 / 29$ & $39 / 21$ & $39 / 21$ & $23 / 37$ & $55 / 5$ & $25 / 35$ \\
\hline $21 / 39$ & $39 / 21$ & $31 / 29$ & $45 / 15$ & $39 / 21$ & $25 / 35$ & $55 / 5$ & $28 / 32$ \\
\hline $21 / 39$ & $45 / 15$ & $31 / 29$ & $55 / 5$ & $39 / 21$ & $28 / 32$ & $55 / 5$ & $29 / 31$ \\
\hline $21 / 39$ & $55 / 5$ & $31 / 29$ & $60 / 0$ & $39 / 21$ & $29 / 31$ & $55 / 5$ & $32 / 28$ \\
\hline $21 / 39$ & $60 / 0$ & $32 / 28$ & $30 / 30$ & $39 / 21$ & $32 / 28$ & $55 / 5$ & $33 / 27$ \\
\hline $23 / 37$ & $37 / 23$ & $32 / 28$ & $31 / 29$ & $39 / 21$ & $33 / 27$ & $55 / 5$ & $37 / 23$ \\
\hline $23 / 37$ & $40 / 20$ & $32 / 28$ & $34 / 26$ & $39 / 21$ & $37 / 23$ & $55 / 5$ & $39 / 21$ \\
\hline $23 / 37$ & $50 / 10$ & $32 / 28$ & $37 / 23$ & $39 / 21$ & $40 / 20$ & $55 / 5$ & $50 / 10$ \\
\hline $23 / 37$ & $57 / 3$ & $32 / 28$ & $40 / 20$ & $39 / 21$ & $50 / 10$ & $55 / 5$ & $57 / 3$ \\
\hline $25 / 35$ & $37 / 23$ & $32 / 28$ & $50 / 10$ & $39 / 21$ & $57 / 3$ & $57 / 3$ & $3 / 57$ \\
\hline $25 / 35$ & $40 / 20$ & $32 / 28$ & $57 / 3$ & $40 / 20$ & $20 / 40$ & $57 / 3$ & $5 / 55$ \\
\hline $25 / 35$ & $50 / 10$ & $33 / 27$ & $28 / 32$ & $40 / 20$ & $21 / 39$ & $57 / 3$ & $20 / 40$ \\
\hline $25 / 35$ & $57 / 3$ & $33 / 27$ & $29 / 31$ & $40 / 20$ & $26 / 34$ & $57 / 3$ & $21 / 39$ \\
\hline $26 / 34$ & $35 / 25$ & $33 / 27$ & $32 / 28$ & $40 / 20$ & $27 / 33$ & $57 / 3$ & $26 / 34$ \\
\hline $26 / 34$ & $39 / 21$ & $33 / 27$ & $34 / 26$ & $40 / 20$ & $30 / 30$ & $57 / 3$ & $27 / 33$ \\
\hline $26 / 34$ & $45 / 15$ & $33 / 27$ & $37 / 23$ & $40 / 20$ & $31 / 29$ & $57 / 3$ & $30 / 30$ \\
\hline $26 / 34$ & $55 / 5$ & $33 / 27$ & $40 / 20$ & $40 / 20$ & $34 / 26$ & $57 / 3$ & $31 / 29$ \\
\hline $26 / 34$ & $60 / 0$ & $33 / 27$ & $50 / 10$ & $40 / 20$ & $35 / 25$ & $57 / 3$ & $34 / 26$ \\
\hline $27 / 33$ & $33 / 27$ & $33 / 27$ & $57 / 3$ & $40 / 20$ & $45 / 15$ & $57 / 3$ & $35 / 25$ \\
\hline $27 / 33$ & $35 / 25$ & $34 / 26$ & $26 / 34$ & $40 / 20$ & $55 / 5$ & $57 / 3$ & $40 / 20$ \\
\hline $27 / 33$ & $39 / 21$ & $34 / 26$ & $27 / 33$ & $40 / 20$ & $60 / 0$ & $57 / 3$ & $45 / 15$ \\
\hline $27 / 33$ & $45 / 15$ & $34 / 26$ & $30 / 30$ & $45 / 15$ & $15 / 45$ & $57 / 3$ & $60 / 0$ \\
\hline $27 / 33$ & $55 / 5$ & $34 / 26$ & $31 / 29$ & $45 / 15$ & $23 / 37$ & $60 / 0$ & $0 / 60$ \\
\hline $27 / 33$ & $60 / 0$ & $34 / 26$ & $35 / 25$ & $45 / 15$ & $25 / 35$ & $60 / 0$ & $10 / 50$ \\
\hline $28 / 32$ & $32 / 28$ & $34 / 26$ & $39 / 21$ & $45 / 15$ & $28 / 32$ & $60 / 0$ & $15 / 45$ \\
\hline $28 / 32$ & $34 / 26$ & $34 / 26$ & $45 / 15$ & $45 / 15$ & $29 / 31$ & $60 / 0$ & $23 / 37$ \\
\hline $28 / 32$ & $37 / 23$ & $34 / 26$ & $55 / 5$ & $45 / 15$ & $32 / 28$ & $60 / 0$ & $25 / 35$ \\
\hline $28 / 32$ & $40 / 20$ & $34 / 26$ & $60 / 0$ & $45 / 15$ & $33 / 27$ & $60 / 0$ & $28 / 32$ \\
\hline $28 / 32$ & $50 / 10$ & $35 / 25$ & $25 / 35$ & $45 / 15$ & $37 / 23$ & $60 / 0$ & $29 / 31$ \\
\hline $28 / 32$ & $57 / 3$ & $35 / 25$ & $28 / 32$ & $45 / 15$ & $39 / 21$ & $60 / 0$ & $32 / 28$ \\
\hline $29 / 31$ & $32 / 28$ & $35 / 25$ & $29 / 31$ & $45 / 15$ & $55 / 5$ & $60 / 0$ & $33 / 27$ \\
\hline $29 / 31$ & $34 / 26$ & $35 / 25$ & $32 / 28$ & $45 / 15$ & $60 / 0$ & $60 / 0$ & $37 / 23$ \\
\hline $29 / 31$ & $37 / 23$ & $35 / 25$ & $33 / 27$ & $50 / 10$ & $20 / 40$ & $60 / 0$ & $39 / 21$ \\
\hline $29 / 31$ & $40 / 20$ & $35 / 25$ & $39 / 21$ & $50 / 10$ & $21 / 39$ & $60 / 0$ & $50 / 10$ \\
\hline $29 / 31$ & $50 / 10$ & $35 / 25$ & $45 / 15$ & $50 / 10$ & $26 / 34$ & $60 / 0$ & $55 / 5$ \\
\hline $29 / 31$ & $57 / 3$ & $35 / 25$ & $55 / 5$ & $50 / 10$ & $27 / 33$ & & \\
\hline $30 / 30$ & $31 / 29$ & $35 / 25$ & $60 / 0$ & $50 / 10$ & $30 / 30$ & & \\
\hline
\end{tabular}

Table 7: Mini-DGs used in the experiment (dictator's/recipients' payoffs). 